\title{
Polaronic quasiparticle picture for generation dynamics of coherent phonons in semiconductors: Transient and nonlinear Fano resonance
}

\author{
Yohei Watanabe, ${ }^{1}$ Ken-ichi Hino, ${ }^{2,3, *}$ Muneaki Hase, ${ }^{4}$ and Nobuya Maeshima ${ }^{2,3}$ \\ ${ }^{1}$ Doctoral Program in Materials Science, Graduate School of Pure and Applied Sciences, University of Tsukuba, \\ Tsukuba, Ibaraki 305-8573, Japan \\ ${ }^{2}$ Division of Materials Science, Faculty of Pure and Applied Sciences, University of Tsukuba, Tsukuba 305-8573, Japan \\ ${ }^{3}$ Center for Computational Sciences, University of Tsukuba, Tsukuba 305-8577, Japan \\ ${ }^{4}$ Division of Applied Physics, Faculty of Pure and Applied Sciences, University of Tsukuba, Tsukuba 305-8573, Japan
}

(Received 15 September 2015; revised manuscript received 7 October 2016; published 3 January 2017)

\begin{abstract}
We examine generation dynamics of coherent phonons in both polar and nonpolar semiconductors, such as GaAs and Si, based on a polaronic-quasiparticle (PQ) model. In this model, the PQ operator is composed of two kinds of operators: one is a quasiboson operator, defined as a linear combination of a set of pairs of electron operators, and the other is a longitudinal optical (LO) phonon operator. In particular, the problem of transient and nonlinear Fano resonance (FR) is tackled, where the vestige of this quantum interference effect was observed exclusively in lightly $n$-doped Si immediately after carriers were excited by an ultrashort pulse laser [M. Hase et al., Nature (London) 426, 51 (2003)], although not observed yet in GaAs. The PQ model enables us to show straightforwardly that the phonon energy state is embedded in continuum states formed by a set of adiabatic eigenstates of the quasiboson; this energy configuration is a necessary condition of the manifestation of the transient FR in the present optically nonlinear system. Numerical calculations are done for photoemission spectra relevant to the retarded longitudinal dielectric function of transient photoexcited states and for power spectra relevant to the LO-phonon displacement function of time. The photoemission spectra show that in undoped $\mathrm{Si}$, an asymmetric spectral profile characteristic of FR comes into existence immediately after the instantaneous carrier excitation to fade out gradually, whereas in undoped GaAs, no asymmetry in spectra appears in the whole temporal region. The similar results are also obtained in the power spectra. These results are in harmony with the reported experimental results. It is found that the obtained difference in spectral profile between undoped $\mathrm{Si}$ and GaAs is attributed to a phase factor of an effective interaction between the LO phonon and the quasiboson. More detailed discussion of the FR dynamics is made in the text.
\end{abstract}

DOI: 10.1103/PhysRevB.95.014301

\section{INTRODUCTION}

The recent progress of laser technology toward the development of ultrashort pulsed laser with high intensity has opened up a new research area exploring ultrafast transitory phenomena governed by strongly photoexcited electronic states in diverse fields of physics and chemistry [1]. The coherent phonon (CP) generation concerned in this study is undoubtedly one of the representative phenomena successfully exposed by virtue of ultrashort pulsed laser; its temporal width is of an order of $10 \mathrm{fs}$, much shorter than a period of a longitudinal optical (LO) phonon [2-4]. Here, the LO-phonon mode is driven at one stroke immediately after the pulse exertion on a crystal to show coherent vibrations. The CPs have been observed in variety of physical systems such as semiconductors [5-15], dielectrics [16-19], semimetals/metals [20-36], high- $T_{c}$ superconductors [37-42], and other materials [43-46]; a great number of other references are cited in Refs. [2,3]. The CP generation dynamics is well described in terms of a classical model following a damped harmonic oscillation, and this is assessed by an initial phase built in the oscillator with an asymptotic sinusoidal form in time [2,3].

There are two classical models hitherto presented for the generation dynamics, namely, the impulsive stimulated Raman scattering (ISRS) model [47-49] and the displacive excitation

*hino@ims.tsukuba.ac.jp of CP (DECP) model [50-52], aside from one more mechanism of ultrafast screening of space-charge/surface-depletion field inherent in polar semiconductors [6,7]. In the ISRS model, the stimulated Raman scattering induced by a pump pulse is considered to govern the generation dynamics, in which the oscillator is driven by an impulsive external force, a deltafunction-like force, associated with Raman polarizability [2,3].

In the DECP model, optical transitions of electrons into excited states alter an equilibrium position of lattice vibration toward a new position, in which the oscillator is driven by a step-function-like external force [2,3]. Further, phenomenological models hybridizing these two models are devised to be made in consistency with experimentally observed initial phases [14,51-54].

Indeed, the classical models mentioned above have successfully revealed overall features of the $\mathrm{CP}$ generation dynamics, but it is the matter of course that a microscopic theory based on the nonequilibrium quantum dynamics is required to bring into light not only details of the dynamics of concern, such as an origin of phenomenological external forces driving CPs, and the related built-in initial phases, but also still unexplored quantum effects. In fact, theoretical studies toward this direction are scarce until now; the density-matrix theory was applied to understand the external forces from the viewpoint of the group theory $[55,56]$, the time-dependent Schrödinger equation was solved numerically to understand an experimentally observed quantum effect [57], the time-dependent density-functional theory was applied to reproduce measured $\mathrm{CP}$ signals in 
Si [58], the Fano-Anderson Hamiltonian [59] was applied to classical dynamics to show the dependence of a Fano resonance (FR) effect [60] on an initial phase [61], and a simple two-level model was applied to CP generation to show the initial-phase dependence on pulse width and detuning [62].

As regards the quantum effect concomitant to the $\mathrm{CP}$ generation, Hase et al. observed the transient FR effect in a lightly $n$-doped Si crystal immediately after carriers were excited by an ultrashort laser pulse [13]. This was considered to result from quantum interference between excited carriers and an LO phonon, which played the roles of continuum and discrete states, respectively. Moreover, the speculation was made that the observed FR showed the evidence of the birth of a polaronic quasiparticle (PQ); this is likely formed in a strongly interacting carrier-LO-phonon system in a moment [63]. It is noted that the transient FR of concern has been observed exclusively in a lightly $n$-doped Si crystal and semimetals/metals such as Bi and Zn [32-35] until now, however, not observed in $p$-doped $\mathrm{Si}$ and GaAs crystals $[11,15]$. In addition, dynamic FR-like interference between Rabi oscillations and $\mathrm{CPs}$ was observed in $\mathrm{CuCl}$ semiconductor microcavities [64].

Thus far, there are a number of theoretical studies regarding these experimental findings. By solving the time-dependent Schrödinger equation in the system of GaAs, Lee et al. [57] calculated a displacement function of $\mathrm{CP}$, and showed that the associated continuous-wavelet transform of this function became the asymmetric shape featuring FR spectra, though apparently opposed to existing experimental results, as mentioned above. The authors further discussed the origin of the resulting FR in a Feynman-diagrammatic manner to conclude that the FR arose from interference between two types of vibrational Raman-scattering processes. Riffe [61] derived the classical Fano oscillator model from the Fano-Anderson Hamiltonian, and showed that Fano's $q$ parameter $q^{(F)}$, which determines the degree of asymmetry of spectra, was incorporated in an initial phase. Misochko and Lebedeva [33] showed the different dependence of $q^{(F)}$ on the initial phase by taking the Fourier transform of the Fano's spectral formula in a direct manner into a temporal region. Further, they confirmed that the experimental results of the $\mathrm{CP}$ signals of $\mathrm{Bi}$ were in harmony with the obtained initial-phase dependence.

This paper is aimed at the following goals. First, one constructs a fully-quantum-mechanical model for the CP generation dynamics available for both polar and nonpolar semiconductors on an equal footing. Given the above-mentioned supposition of the PQ $[13,63]$, a PQ operator is introduced to this model, which is defined as composed of two kinds of operators: one is a quasiboson operator, given by a linear combination of a set of pairs of electron operators, and the other is an LO-phonon operator. Second, based on this PQ model, one tracks the origin of the manifestation of the transient FR in the early stage of the whole CP dynamics. This temporal region is defined as the region during which a great number of carriers still stay in excited states and the quantum processes govern the $\mathrm{CP}$ dynamics, and this will be termed hereafter as the early time region. For this purpose, one examines a retarded longitudinal susceptibility leading to induced charge density and photoemission spectra, and an LO-phonon displacement function related to experimental observables in the systems of undoped Si and undoped GaAs. It is noted that any delayed formation of LO-phonon plasmon coupled modes in undoped GaAs is not taken into account throughout this study since these modes are not created instantaneously in the early-time region [3,65-73]. Third, the obtained results are compared to the reported studies with discussion $[13,57,61]$.

It is pointed out that there is a different type of FR from the present one in semiconductors, which is generated by incoherent Raman scattering driven by irradiation of a continuous-wave (cw) laser [67,74-91]. This FR process was observed in the system of heavily $p$-doped $\mathrm{Si}$ in the $\Gamma$ point $[67,79,81,83,85,88]$ and heavily $n$-doped $\mathrm{Si}$ in the $X$ valley [84]. It is interesting that the manner of doping has an effect on a sign of the resulting Fano's $q$ parameter; this parameter tends to be positive (negative) in the $p(n)$-doping case, showing FR spectra with a dip (peak), followed by a peak (dip) [88]. In addition, the FR was also discerned in the system of $\delta$-doped GaAs [89-91].

As is well known, FR is considered as one of the fundamental concepts common to diverse fields of physics [92]. In particular, within the restriction just to the FR processes triggered by laser irradiation, the FR of concern is regarded as unusual transient and optically nonlinear processes, considerably distinct from most of FR processes observed in linear optical processes due to cw-laser irradiation [93]. Actually, the present FR is caused by strongly photoexcited carriers generated by a short pulsed laser, and is short lived enough to appear just immediately after the completion of the pulse irradiation.

The number of studies pertaining to this type of FR is really limited due presumably to difficulties of experimental measurements and theoretical predictions in advance. One example belonging to this type is a transient excitonic FR manifesting itself in ultrafast optical processes [94-97]. Precisely for this reason, it is expected that the studies directed toward this type of FR have the potential to explore a new area of research with enriched physics. Hereafter, the FR of concern is termed as transient FR just for the sake of simplicity unless otherwise stated, although this should be referred to as transient and nonlinear FR, as titled in this paper.

The remainder of this paper is organized as follows. Section II describes the theoretical framework, Sec. III presents the results and discussion, and Sec. IV presents the conclusion. Atomic units (a.u.) are used throughout unless otherwise stated.

\section{THEORY}

\section{A. Introduction of polaronic quasiparticle operators}

\section{Hamiltonian of the system}

The total Hamiltonian $\hat{H}$ of the present system is given as follows:

$$
\hat{H}=\hat{H}_{e}+\hat{H}^{\prime}(t)+\hat{H}_{p}+\hat{H}_{e-p},
$$

where $\hat{H}_{e}, \hat{H}^{\prime}(t), \hat{H}_{p}$, and $\hat{H}_{e-p}$ represent an electron Hamiltonian, an interaction Hamiltonian between laser and electron at time $t$, an LO-phonon Hamiltonian, and an interaction Hamiltonian between electron and phonon, respectively. $\hat{H}_{e}$ 
is provided within a two-band model just taking account of the energetically lowest conduction band $(c)$ and the energetically highest valence band $(v)$ as follows:

$$
\hat{H}_{e}=\sum_{b(=c, v), \boldsymbol{k}} \varepsilon_{b \boldsymbol{k}}^{\prime} a_{b \boldsymbol{k}}^{\dagger} a_{b \boldsymbol{k}}+\frac{1}{2} \sum_{\boldsymbol{q}(\neq \mathbf{0})} V_{\boldsymbol{q}}^{(C)} \hat{\rho}_{\boldsymbol{q}} \hat{\rho}_{-\boldsymbol{q}},
$$

where $a_{b \boldsymbol{k}}^{\dagger}\left(a_{b \boldsymbol{k}}\right)$ represents a creation (annihilation) operator of electron with $b$ and $\boldsymbol{k}$ as a band index and Bloch momentum, respectively. Hereafter, it is understood that a spin index of electron is suppressed in the notation of $\boldsymbol{k}$. Further, $V_{\boldsymbol{q}}^{(C)}$ represents a Coulomb potential given by

$$
V_{\boldsymbol{q}}^{(C)}=\frac{4 \pi}{\epsilon_{\infty} V} \frac{1}{\boldsymbol{q}^{2}}
$$

with $\boldsymbol{q}, V$, and $\epsilon_{\infty}$ as momentum, volume of crystal, and a dielectric constant in the high-frequency limit, respectively, $\hat{\rho}_{\boldsymbol{q}}$ is defined as

$$
\hat{\rho}_{\boldsymbol{q}}=\sum_{b, \boldsymbol{k}} a_{b, \boldsymbol{k}+\boldsymbol{q}}^{\dagger} a_{b \boldsymbol{k}}
$$

and $\varepsilon_{b \boldsymbol{k}}^{\prime}$ is given by

$$
\varepsilon_{b \boldsymbol{k}}^{\prime}=\varepsilon_{b \boldsymbol{k}}-\frac{1}{2} \sum_{\boldsymbol{q}(\neq \mathbf{0})} V_{\boldsymbol{q}}^{(C)},
$$

where $\varepsilon_{b \boldsymbol{k}}$ represents an energy dispersion of $b$-band electron. $\hat{H}^{\prime}(t)$ is expressed as

$$
\hat{H}^{\prime}(t)=-\frac{1}{2} \sum_{b, b^{\prime}(\neq b), \boldsymbol{k}}\left[\Omega_{b b^{\prime}}(t) a_{b \boldsymbol{k}}^{\dagger} a_{b^{\prime} \boldsymbol{k}}+\Omega_{b b^{\prime}}^{*}(t) a_{b^{\prime} \boldsymbol{k}}^{\dagger} a_{b \boldsymbol{k}}\right],
$$

where $\Omega_{b b^{\prime}}(t)=d_{b b^{\prime}} F(t)$ with $F(t)$ an electric field of an irradiated pump laser and $d_{b b^{\prime}}$ an electric dipole moment between $b$ and $b^{\prime}$ bands; slight $\boldsymbol{k}$ dependence of the dipole moment is neglected. Further, a factor of $\frac{1}{2}$ in front of the summation of the right-hand side implies that the summations with respect to both $b$ and $b^{\prime}$ are taken over all of bands concerned here. $\hat{H}_{p}$ is given by

$$
\hat{H}_{p}=\sum_{\boldsymbol{q}} \omega_{\boldsymbol{q}}^{(\mathrm{LO})} c_{\boldsymbol{q}}^{\dagger} c_{\boldsymbol{q}},
$$

where $c_{\boldsymbol{q}}^{\dagger}\left(c_{\boldsymbol{q}}\right)$ represents a creation (annihilation) operator of LO phonon with an energy dispersion $\omega_{q}^{(\mathrm{LO})}$. The zero-point energy of the phonon is omitted just for the sake of simplicity. Further, $\hat{H}_{e-p}$ is given by

$$
\hat{H}_{e-p}=\sum_{b, \boldsymbol{q}, \boldsymbol{k}}\left(g_{b \boldsymbol{q}} c_{\boldsymbol{q}} a_{b \boldsymbol{k}+\boldsymbol{q}}^{\dagger} a_{b \boldsymbol{k}}+g_{b \boldsymbol{q}}^{*} c_{\boldsymbol{q}}^{\dagger} a_{b \boldsymbol{k}}^{\dagger} a_{b \boldsymbol{k}+\boldsymbol{q}}\right),
$$

where $g_{b q}$ is a coupling constant of $b$-band electron with an LO phonon.

\section{Quasiboson operators}

We take into consideration the equation of motion for a composite operator $A_{\boldsymbol{q}}^{\dagger}\left(\boldsymbol{k} b b^{\prime}\right)$ defined by

$$
A_{q}^{\dagger}\left(\boldsymbol{k} b b^{\prime}\right)=a_{b, \boldsymbol{k}+\boldsymbol{q}}^{\dagger} a_{b^{\prime} \boldsymbol{k}},
$$

which is provided in terms of the Heisenberg equation as

$$
\begin{aligned}
- & i\left(\frac{d}{d t}+\frac{1}{T_{\boldsymbol{q} \boldsymbol{k} b b^{\prime}}}\right) A_{\boldsymbol{q}}^{\dagger}\left(\boldsymbol{k} b b^{\prime}\right) \\
& =\left[\hat{\mathcal{H}}_{e}(t), A_{\boldsymbol{q}}^{\dagger}\left(\boldsymbol{k} b b^{\prime}\right)\right]+\left[\hat{H}_{e-p}, A_{\boldsymbol{q}}^{\dagger}\left(\boldsymbol{k} b b^{\prime}\right)\right],
\end{aligned}
$$

where an electronic Hamiltonian $\hat{\mathcal{H}}_{e}(t)$ is defined as

$$
\hat{\mathcal{H}}_{e}(t)=\hat{H}_{e}+\hat{H}^{\prime}(t) \text {. }
$$

Further, $T_{q k b b^{\prime}}$ represents a phenomenological relaxation-time constant of induced carrier density $A_{\boldsymbol{q}}^{\dagger}\left(\boldsymbol{k} b b^{\prime}\right)$ with anisotropic momentum distribution. It is noted that the degree of spatial anisotropy shown by $|\boldsymbol{q}|$ is finite here, even though it is quite small: $\boldsymbol{q} \neq \mathbf{0}$.

The first commutator in the right-hand side of Eq. (10) is evaluated by making a factorization approximation to split four operator terms such as $a_{\tilde{b}, \tilde{\boldsymbol{k}}+\tilde{\boldsymbol{q}}}^{\dagger} a_{\tilde{b}^{\prime} \tilde{\boldsymbol{k}}} a_{b, \boldsymbol{k}+\boldsymbol{q}}^{\dagger} a_{b^{\prime} \boldsymbol{k}}$ into a product of the operator $A_{\tilde{\boldsymbol{q}}}^{\dagger}\left(\tilde{\boldsymbol{k}} \tilde{b} \tilde{b}^{\prime}\right)$ and a single-particle density matrix $\rho_{b b^{\prime} \boldsymbol{k}} \equiv\left\langle a_{b, \boldsymbol{k}}^{\dagger} a_{b^{\prime} \boldsymbol{k}}\right\rangle$, where $\langle\hat{X}\rangle$ means an expectation value of operator $\hat{X}$ with respect to the ground state. The following expression is obtained:

$$
\left[\hat{\mathcal{H}}_{e}(t), A_{\boldsymbol{q}}^{\dagger}\left(\boldsymbol{k} b b^{\prime}\right)\right] \approx \sum_{\tilde{\boldsymbol{k}} \tilde{b} \tilde{b}^{\prime}} A_{\boldsymbol{q}}^{\dagger}\left(\tilde{\boldsymbol{k}} \tilde{b} \tilde{b}^{\prime}\right) Z_{\boldsymbol{q}}\left(\tilde{\boldsymbol{k}} \tilde{b} \tilde{b}^{\prime}, \boldsymbol{k} b b^{\prime}\right),
$$

where $Z_{q}$ is a $c$-number non-Hermitian matrix, the explicit expression of which is given by

$$
\begin{aligned}
& Z_{\boldsymbol{q}}\left(\boldsymbol{k}_{1} b_{1} b_{1}^{\prime}, \boldsymbol{k}_{2} b_{2} b_{2}^{\prime}\right) \\
& =w_{b_{1} b_{1}^{\prime} \boldsymbol{k}_{1} \boldsymbol{q}} \delta_{b_{1} b_{2}} \delta_{b_{1}^{\prime} b_{2}^{\prime}} \delta_{\boldsymbol{k}_{1} \boldsymbol{k}_{2}}+V_{\boldsymbol{q}}^{(C)} \delta_{b_{1} b_{1}^{\prime}} \Delta \rho_{b_{2} b_{2}^{\prime} \boldsymbol{k}_{2} \boldsymbol{q}} \\
& \quad-\Omega_{b_{1} \bar{b}_{1} \boldsymbol{k}_{1}}^{(R)} \delta_{b_{1} \bar{b}_{2}} \delta_{b_{1}^{\prime} b_{2}^{\prime}} \delta_{\boldsymbol{k}_{1} \boldsymbol{k}_{2}}+\Omega_{\bar{b}_{1}^{\prime} b_{1}^{\prime} \boldsymbol{k}_{1}}^{(R)} \delta_{b_{1}^{\prime} \bar{b}_{2}^{\prime}} \delta_{b_{1} b_{2}} \delta_{\boldsymbol{k}_{1} \boldsymbol{k}_{2}},
\end{aligned}
$$

where the barred index $\bar{b}$ means the index that is not equal to $b$, that is, $\bar{c}=v$ and $\bar{v}=c$. In Eq. (13),

$$
w_{b b^{\prime} \boldsymbol{k} \boldsymbol{q}}=\varepsilon_{b \boldsymbol{k}+\boldsymbol{q}}^{(r)}-\varepsilon_{b^{\prime} \boldsymbol{k}}^{(r)},
$$

where $\varepsilon_{b \boldsymbol{k}}^{(r)}$ represents a renormalized $b$-band electron energy given by [98]

$$
\varepsilon_{b \boldsymbol{k}}^{(r)}=\varepsilon_{b \boldsymbol{k}}^{\prime}-\sum_{\boldsymbol{q}} V_{\boldsymbol{q}}^{(C)} \rho_{b b \boldsymbol{k}+\boldsymbol{q}},
$$

$\Omega_{b b^{\prime} \boldsymbol{k}}^{(R)}$ represents a Rabi frequency given by [98]

$$
\Omega_{b b^{\prime} \boldsymbol{k}}^{(R)}=\Omega_{b b^{\prime}}+\sum_{\boldsymbol{q}} V_{\boldsymbol{q}}^{(C)} \rho_{b b^{\prime} \boldsymbol{k}+\boldsymbol{q}},
$$

and

$$
\Delta \rho_{b b^{\prime} \boldsymbol{k} \boldsymbol{q}}=\rho_{b b^{\prime} \boldsymbol{k}}-\rho_{b b^{\prime} \boldsymbol{k}+\boldsymbol{q}} .
$$

$F(t)$ is expressed as

$$
F(t)=F_{0}(t) \cos \omega_{0} t,
$$

where $\omega_{0}$ represents center frequency of the laser field, and $F_{0}(t)$ represents an envelope with narrow temporal width $\tau_{L}$ satisfying $\tau_{L} \ll 2 \pi / \omega_{q}^{(\mathrm{LO})}$; in the physical systems of concern, $\tau_{L}$ is of the order of a couple of tens of femtoseconds at most. 
Hereafter, let $F_{0}(t)$ be of squared shape just for the sake of simplicity, that is,

$$
F_{0}(t)=\mathcal{F}_{0} \theta\left(t+\tau_{L} / 2\right) \theta\left(\tau_{L} / 2-t\right)
$$

with $\mathcal{F}_{0}$ constant. It is convenient to remove from Eq. (10) high-frequency contributions by means of a well-justified rotational-wave approximation [99]. This is done by replacing $A_{\boldsymbol{q}}^{\dagger}\left(\boldsymbol{k} b b^{\prime}\right)$ and $\rho_{b b^{\prime} \boldsymbol{k}}$ in Eq. (10) by $e^{i \bar{\omega}_{b b^{\prime}} t} \bar{A}_{\boldsymbol{q}}^{\dagger}\left(\boldsymbol{k} b b^{\prime}\right)$ and $e^{i \bar{\omega}_{b b^{\prime}} t} \bar{\rho}_{b b^{\prime} k}$, respectively, where $\bar{\omega}_{c v}=\omega_{0}, \bar{\omega}_{v c}=-\omega_{0}$, and $\bar{\omega}_{b b}=0$. Thus, Eq. (10) is cast into the form

$$
\begin{aligned}
-i & \left(\frac{d}{d t}+\frac{1}{T_{\boldsymbol{q} \boldsymbol{k} b b^{\prime}}}\right) \bar{A}_{\boldsymbol{q}}^{\dagger}\left(\boldsymbol{k} b b^{\prime}\right) \\
= & {\left[\hat{\mathcal{H}}_{e}(t), \bar{A}_{\boldsymbol{q}}^{\dagger}\left(\boldsymbol{k} b b^{\prime}\right)\right]-\bar{A}_{\boldsymbol{q}}^{\dagger}\left(\boldsymbol{k} b b^{\prime}\right) \bar{\omega}_{b b^{\prime}}+\left[\hat{H}_{e-p}, \bar{A}_{\boldsymbol{q}}^{\dagger}\left(\boldsymbol{k} b b^{\prime}\right)\right] } \\
& \approx \sum_{\tilde{\boldsymbol{k}} \tilde{b} \tilde{b}^{\prime}} \bar{A}_{\boldsymbol{q}}^{\dagger}\left(\tilde{\boldsymbol{k}} \tilde{b} \tilde{b}^{\prime}\right) \bar{Z}_{\boldsymbol{q}}\left(\tilde{\boldsymbol{k}} \tilde{b} \tilde{b}^{\prime}, \boldsymbol{k} b b^{\prime}\right)+\left[\hat{H}_{e-p}, \bar{A}_{\boldsymbol{q}}^{\dagger}\left(\boldsymbol{k} b b^{\prime}\right)\right],
\end{aligned}
$$

where $\bar{Z}_{\boldsymbol{q}}\left(\tilde{\boldsymbol{k}} \tilde{b} \tilde{b}^{\prime}, \boldsymbol{k} b b^{\prime}\right)$ is provided from $Z_{\boldsymbol{q}}\left(\tilde{\boldsymbol{k}} \tilde{b} \tilde{b}^{\prime}, \boldsymbol{k} b b^{\prime}\right)$ of Eq. (13) by replacing $\rho_{b b^{\prime} \boldsymbol{k}}$ by $\bar{\rho}_{b b^{\prime} \boldsymbol{k}}, \Delta \rho_{b b^{\prime} \boldsymbol{k} \boldsymbol{q}}$ by $\Delta \bar{\rho}_{b b^{\prime} \boldsymbol{k} \boldsymbol{q}}, w_{b b^{\prime} \boldsymbol{k} \boldsymbol{q}}$ by $\bar{w}_{b b^{\prime} \boldsymbol{k} \boldsymbol{q}} \equiv w_{b b^{\prime} \boldsymbol{k} \boldsymbol{q}}-\bar{\omega}_{b b^{\prime}}$, and $\Omega_{b b^{\prime} \boldsymbol{k}}^{(R)}$ by

$$
\bar{\Omega}_{b b^{\prime} \boldsymbol{k}}^{(R)}=\frac{1}{2} \Omega_{0 b b^{\prime}}+\sum_{\boldsymbol{q}} V_{\boldsymbol{q}}^{(C)} \bar{\rho}_{b b^{\prime} \boldsymbol{k}+\boldsymbol{q}}
$$

with $\Omega_{0 b b^{\prime}}(t)=d_{b b^{\prime}} F_{0}(t) \delta_{b^{\prime} \bar{b}}$.

The non-Hermitian matrix $\bar{Z}_{\boldsymbol{q}}$ is a slowly varying function in time since rapidly time-varying contributions are removed in Eq. (20) owing to the rotational-wave approximation, aside from the discontinuity at $t= \pm \tau_{L} / 2$ due to Eq. (19). Bearing in mind this situation, we tackle left and right eigenvalue problems of $\bar{Z}_{\boldsymbol{q}}[100,101]$. These problems are described by

$$
U_{q}^{L \dagger} \bar{Z}_{q}=\mathcal{E}_{q} U_{q}^{L \dagger}
$$

and

$$
\bar{Z}_{\boldsymbol{q}} U_{\boldsymbol{q}}^{R}=U_{\boldsymbol{q}}^{R} \mathcal{E}_{\boldsymbol{q}}
$$

respectively, in terms of an adiabatic-eigenvalue diagonalmatrix $\mathcal{E}_{\boldsymbol{q}}$, and the associated biorthogonal set of adiabatic eigenvectors $\left\{U_{\boldsymbol{q}}^{L}, U_{\boldsymbol{q}}^{R}\right\}$ with time $t$ fixed as a parameter; the orthogonality relation and the completeness are read as $U_{\boldsymbol{q}}^{L \dagger} U_{\boldsymbol{q}}^{R}=1$ and $U_{\boldsymbol{q}}^{R} U_{\boldsymbol{q}}^{L \dagger}=1$, respectively [100,101]. Here, matrix notations are employed, that is, $\bar{Z}_{\boldsymbol{q}}=\left\{\bar{Z}_{\boldsymbol{q}}\left(\tilde{\boldsymbol{k}} \tilde{b} \tilde{b}^{\prime}, \boldsymbol{k} b b^{\prime}\right)\right\}$, $\mathcal{E}_{\boldsymbol{q}}=\left\{\mathcal{E}_{\boldsymbol{q} \alpha}\right\}$, and $U_{\boldsymbol{q}}^{L / R}=\left\{U_{\boldsymbol{q} \alpha}^{L / R}\left(\boldsymbol{k} b b^{\prime}\right)\right\}$, where $\mathcal{E}_{\boldsymbol{q} \alpha}$ and $U_{\boldsymbol{q} \alpha}^{L / R}\left(\boldsymbol{k} b b^{\prime}\right)$ are the $\alpha$ th eigenvalue and eigenvector, respectively. Similarly to $\bar{Z}_{\boldsymbol{q}}$, both of $\mathcal{E}_{\boldsymbol{q}}$ and $U_{\boldsymbol{q}}^{L / R}$ are slowly varying functions in time except at $t= \pm \tau_{L} / 2$. Equations (22) and (23) can be solved in an analytic manner, as shown in Ref. [102].

Given the relation $\bar{Z}_{\boldsymbol{q}}=U_{\boldsymbol{q}}^{R} \mathcal{E}_{\boldsymbol{q}} U_{\boldsymbol{q}}^{L \dagger}$, Eq. (20) is recast into the form

$$
-i \frac{d B_{\boldsymbol{q} \alpha}^{\dagger}}{d t}=B_{\boldsymbol{q} \alpha}^{\dagger} \mathcal{E}_{\boldsymbol{q} \alpha}+i \sum_{\alpha^{\prime}} B_{\boldsymbol{q} \alpha^{\prime}}^{\dagger} \mathcal{W}_{\boldsymbol{q} \alpha^{\prime} \alpha}+\left[\hat{H}_{e-p}, B_{\boldsymbol{q} \alpha}^{\dagger}\right]
$$

Here, the operator $B_{\boldsymbol{q} \alpha}^{\dagger}$ is defined as

$$
B_{\boldsymbol{q} \alpha}^{\dagger}=\sum_{\boldsymbol{k} b b^{\prime}} \bar{A}_{\boldsymbol{q}}^{\dagger}\left(\boldsymbol{k} b b^{\prime}\right) U_{\boldsymbol{q} \alpha}^{R}\left(\boldsymbol{k} b b^{\prime}\right) \equiv \bar{A}_{\boldsymbol{q}}^{\dagger} U_{\boldsymbol{q} \alpha}^{R}
$$

and

$$
\mathcal{W}_{\boldsymbol{q} \alpha^{\prime} \alpha}=W_{\boldsymbol{q} \alpha^{\prime} \alpha}+\frac{\gamma_{\boldsymbol{q} \alpha^{\prime} \alpha}^{(B)}}{2},
$$

where a nonadiabatic coupling between $\alpha^{\prime}$ and $\alpha$ is represented as

$$
W_{\boldsymbol{q} \alpha^{\prime} \alpha}=\sum_{\boldsymbol{k} b b^{\prime}} \frac{d U_{\boldsymbol{q} \alpha^{\prime}}^{L \dagger}\left(\boldsymbol{k} b b^{\prime}\right)}{d t} U_{\boldsymbol{q} \alpha}^{R}\left(\boldsymbol{k} b b^{\prime}\right)
$$

and

$$
\frac{\gamma_{\boldsymbol{q} \alpha^{\prime} \alpha}^{(B)}}{2}=\sum_{\boldsymbol{k} b b^{\prime}} U_{\boldsymbol{q} \alpha^{\prime}}^{L \dagger}\left(\boldsymbol{k} b b^{\prime}\right) \frac{1}{T_{\boldsymbol{q} \boldsymbol{k} b b^{\prime}}} U_{\boldsymbol{q} \alpha}^{R}\left(\boldsymbol{k} b b^{\prime}\right) .
$$

Equation (24) is nothing but adiabatic coupled equations, where $\mathcal{E}_{\boldsymbol{q} \alpha}(t)$ is adiabatic energy at time $t$ associated with the operator $B_{q \alpha}^{\dagger}(t)$ thus introduced. Hereafter, this operator is termed as a creation operator of quasiboson, and the corresponding annihilation operator is defined as

$$
B_{\boldsymbol{q} \alpha}=\sum_{\boldsymbol{k} b b^{\prime}} U_{\boldsymbol{q} \alpha}^{R \dagger}\left(\boldsymbol{k} b b^{\prime}\right) \bar{A}_{\boldsymbol{q}}\left(\boldsymbol{k} b b^{\prime}\right) \equiv U_{\boldsymbol{q} \alpha}^{R^{\dagger}} \bar{A}_{\boldsymbol{q}} .
$$

The quasibosonization scheme developed here is supplemented in more detail in Ref. [103]. It is noted that $B_{q \alpha}(t)$ and $B_{q \alpha}^{\dagger}(t)$ do not satisfy the equal-time commutation relations for a real boson, that is, $\left[B_{\boldsymbol{q} \alpha}(t), B_{\boldsymbol{q}^{\prime} \alpha^{\prime}}^{\dagger}(t)\right] \neq \delta_{\boldsymbol{q} \boldsymbol{q}^{\prime}} \delta_{\alpha \alpha^{\prime}}$, and that $\mathcal{E}_{\boldsymbol{q} \alpha}$ is a complex number in general, even though $B_{q \alpha}^{\dagger}$ is Hermitian conjugate of $B_{q \alpha}$. The set of eigenstates $\{\alpha\}$ is composed of continuum states represented as $\beta$ with eigenenergy $\mathcal{E}_{\boldsymbol{q} \beta}$ and a single discrete energy state represented as $\alpha_{1}$ with eigenenergy $\mathcal{E}_{\boldsymbol{q} \alpha_{1}}:\{\alpha\}=\left(\{\beta\}, \alpha_{1}\right)$. As shown later in Sec. III A, the state $\beta$ corresponds to electron-hole continuum arising from interband transitions, and the state $\alpha_{1}$ corresponds to a plasmonlike mode.

Following Eqs. (8), (25), and (29), $\hat{H}_{e-p}$ is rewritten as

$$
\hat{H}_{e-p}=\sum_{\boldsymbol{q}, \alpha}\left(M_{\boldsymbol{q} \alpha} c_{\boldsymbol{q}} B_{\boldsymbol{q} \alpha}^{\dagger}+M_{\boldsymbol{q} \alpha}^{*} c_{\boldsymbol{q}}^{\dagger} B_{\boldsymbol{q} \alpha}\right)
$$

where an effective coupling between quasiboson and LO phonon becomes of the form

$$
M_{\boldsymbol{q} \alpha}=\sum_{\boldsymbol{k} b} g_{b \boldsymbol{q}} U_{\boldsymbol{q} \alpha}^{L \dagger}(\boldsymbol{k} b b) .
$$

The commutator of $\left[\hat{H}_{e-p}, B_{\boldsymbol{q} \alpha}^{\dagger}\right]$ in Eq. (24) is evaluated by employing the factorization approximation, by which $\left[B_{\boldsymbol{q} \alpha}, B_{\boldsymbol{q}^{\prime} \alpha^{\prime}}^{\dagger}\right]$ and $\left[B_{\boldsymbol{q}^{\alpha}}^{\dagger}, B_{\boldsymbol{q}^{\prime} \alpha^{\prime}}^{\dagger}\right]$ are replaced by $\left\langle\left[B_{\boldsymbol{q} \alpha}, B_{\boldsymbol{q}^{\prime} \alpha^{\prime}}^{\dagger}\right]\right\rangle$ and $\left\langle\left[B_{q_{\alpha} \alpha}^{\dagger}, B_{q^{\prime} \alpha^{\prime}}^{\dagger}\right]\right\rangle$, respectively. Thus, this leads to

$$
\left[\hat{H}_{e-p}, B_{q \alpha}^{\dagger}\right] \approx M_{-q \alpha}^{\prime \prime} c_{-q}+M_{q \alpha}^{\prime *} c_{q}^{\dagger},
$$

where

$$
\begin{aligned}
M_{-\boldsymbol{q} \alpha}^{\prime \prime} & =\sum_{\alpha^{\prime}} M_{-\boldsymbol{q} \alpha^{\prime}}\left\langle\left[B_{-\boldsymbol{q} \alpha^{\prime}}^{\dagger}, B_{\boldsymbol{q} \alpha}^{\dagger}\right]\right\rangle \\
& =\sum_{\boldsymbol{k} b b^{\prime}}\left(g_{b-\boldsymbol{q}} \bar{\rho}_{b b^{\prime} \boldsymbol{k}}-g_{b^{\prime}-\boldsymbol{q}} \bar{\rho}_{b b^{\prime} \boldsymbol{k}+\boldsymbol{q}}\right) U_{\boldsymbol{q} \alpha}^{R}\left(\boldsymbol{k} b b^{\prime}\right)
\end{aligned}
$$


and

$$
\begin{aligned}
M_{\boldsymbol{q} \alpha}^{\prime *} & =\sum_{\alpha^{\prime}} M_{\boldsymbol{q} \alpha^{\prime}}^{*}\left\langle\left[B_{\boldsymbol{q} \alpha^{\prime}}, B_{\boldsymbol{q} \alpha}^{\dagger}\right]\right\rangle \\
& =\sum_{\boldsymbol{k} b b^{\prime}}\left(g_{b \boldsymbol{q}}^{*} \bar{\rho}_{b b^{\prime} \boldsymbol{k}}-g_{b^{\prime} \boldsymbol{q}}^{*} \bar{\rho}_{b b^{\prime} \boldsymbol{k}+\boldsymbol{q}}\right) U_{\boldsymbol{q} \alpha}^{R}\left(\boldsymbol{k} b b^{\prime}\right) .
\end{aligned}
$$

It is noted that $M_{\boldsymbol{q} \alpha}, M_{\boldsymbol{q} \alpha}^{\prime}$, and $M_{-\boldsymbol{q} \alpha}^{\prime \prime}$ are slowly varying functions in time except at $t= \pm \tau_{L} / 2$ since all of these functions are represented in terms of the adiabatic eigenvectors $U_{\boldsymbol{q}}^{L^{\dagger}}$ and $U_{\boldsymbol{q}}^{R}$ and the slowly time-varying density matrices $\bar{\rho}_{b b^{\prime} \boldsymbol{k}}$. This fact is a cardinal point that gives a theoretical basis to the introduction of the PQ picture aimed at in this study.

\section{Polaronic quasiparticle operators}

Consulting Eq. (32), the adiabatic coupled equations given by Eq. (24) become of the form

$$
\begin{aligned}
-i \frac{d B_{\boldsymbol{q} \alpha}^{\dagger}}{d t}= & B_{\boldsymbol{q} \alpha}^{\dagger} \mathcal{E}_{\boldsymbol{q} \alpha}+c_{\boldsymbol{q}}^{\dagger} M_{\boldsymbol{q} \alpha}^{\prime *} \\
& +i \sum_{\alpha^{\prime}} B_{\boldsymbol{q} \alpha^{\prime}}^{\dagger} \mathcal{W}_{\boldsymbol{q} \alpha^{\prime} \alpha}+M_{-\boldsymbol{q} \alpha}^{\prime \prime} c_{-\boldsymbol{q}}
\end{aligned}
$$

On the other hand, the equation of motion of the LO phonon is described by

$$
-i \frac{d c_{\boldsymbol{q}}^{\dagger}}{d t}=\sum_{\alpha} B_{\boldsymbol{q} \alpha}^{\dagger} M_{\boldsymbol{q} \alpha}+c_{\boldsymbol{q}}^{\dagger} \omega_{\boldsymbol{q}}^{(\mathrm{LO})} .
$$

Both of Eqs. (35) and (36) are integrated into a single equation in terms of matrix notations as follows:

$$
\begin{aligned}
-i \frac{d}{d t}\left(B_{\boldsymbol{q}}^{\dagger}, c_{\boldsymbol{q}}^{\dagger}\right)= & \left(B_{\boldsymbol{q}}^{\dagger}, c_{\boldsymbol{q}}^{\dagger}\right) h_{\boldsymbol{q}} \\
& +\left(i B_{\boldsymbol{q}}^{\dagger} \mathcal{W}_{\boldsymbol{q}}+M_{-\boldsymbol{q}}^{\prime \prime} c_{-\boldsymbol{q}}, 0\right) .
\end{aligned}
$$

Here, $h_{\boldsymbol{q}} \equiv\left\{h_{\boldsymbol{q} \gamma \gamma^{\prime}}\right\}$ is a non-Hermitian matrix given by

$$
h_{q}=\left(\begin{array}{cc}
\mathcal{E}_{q} & M_{q} \\
M_{q}^{\prime \dagger} & \omega_{q}^{(\mathrm{LO})}
\end{array}\right),
$$

with $\gamma, \gamma^{\prime}=1 \sim N+2$, where $N$ represents the number of electron-hole (discretized) continua, namely, $\beta=1 \sim N$, aside from two discrete states attributed to a plasmonlike mode and an LO-phonon mode designated by $\alpha_{1}$ and $\alpha_{2}$, respectively: $\{\gamma\}=\left(\{\beta\}, \alpha_{1}, \alpha_{2}\right)$. It is understood that the matrix indices of $\alpha^{\prime}, \beta^{\prime}$, and $\gamma^{\prime}$ will be adopted with the same meanings as $\alpha, \beta$, and $\gamma$, respectively. The same rule holds for $\alpha_{i}^{\prime}(i=1,2)$.

In this study, the case is exclusively examined that both of the discrete levels of $\alpha_{1}$ and $\alpha_{2}$ are embedded into the continua $\{\beta\}$; the reason for which will be stated in more detail in Sec. III A. This case belongs to the Fano problem, namely, the multichannel scattering problem with one open channel and two closed channels, aside from $h_{\boldsymbol{q}}$ being non-Hermitian. Thus, the following coupled equations are taken account of:

$$
\sum_{\gamma^{\prime}} h_{\boldsymbol{q} \gamma \gamma^{\prime}} V_{\boldsymbol{q} \gamma^{\prime} \beta}^{R}=V_{\boldsymbol{q} \gamma \beta}^{R} \mathcal{E}_{\boldsymbol{q} \beta},
$$

where $V_{\boldsymbol{q} \beta}^{R}=\left\{V_{\boldsymbol{q} \gamma \beta}^{R}\right\}$ is the right vector showing the solution for given energy $\mathcal{E}_{\boldsymbol{q} \beta}$.
In terms of this vector, a set of $N$ operators $F_{q \beta}^{\dagger}(\beta=1 \sim$ $N)$ are defined as

$$
F_{\boldsymbol{q} \beta}^{\dagger}=\sum_{\beta^{\prime}} B_{\boldsymbol{q} \beta^{\prime}}^{\dagger} V_{\boldsymbol{q} \beta^{\prime} \beta}^{R}+B_{\boldsymbol{q} \alpha_{1}}^{\dagger} V_{\boldsymbol{q} \alpha_{1} \beta}^{R}+c_{\boldsymbol{q}}^{\dagger} V_{\boldsymbol{q} \alpha_{2} \beta}^{R} .
$$

Further, the left vector $V_{\boldsymbol{q} \beta}^{L \dagger}=\left\{V_{\boldsymbol{q} \beta \gamma}^{L \dagger}\right\}$ associated with $V_{\boldsymbol{q} \beta}^{R}$ is introduced to ensure the inverse relation

$$
B_{\boldsymbol{q} \alpha}^{\dagger}=\sum_{\beta} F_{\boldsymbol{q} \beta}^{\dagger} V_{\boldsymbol{q} \beta \alpha}^{L \dagger}, \quad c_{\boldsymbol{q}}^{\dagger}=\sum_{\beta} F_{\boldsymbol{q} \beta}^{\dagger} V_{\boldsymbol{q} \beta \alpha_{2}}^{L \dagger},
$$

where $\sum_{\gamma} V_{\boldsymbol{q} \beta \gamma}^{L \dagger} V_{\boldsymbol{q} \gamma \beta^{\prime}}^{R}=\delta_{\beta \beta^{\prime}}$ and $\sum_{\beta} V_{\boldsymbol{q} \gamma \beta}^{R} V_{\boldsymbol{q} \beta \gamma^{\prime}}^{L \dagger}=\delta_{\gamma \gamma^{\prime}}$.

Given Eq. (40), Eq. (37) becomes adiabatic coupled equations for $F_{q}^{\dagger}$ :

$$
\begin{aligned}
-i \frac{d}{d t} F_{\boldsymbol{q} \beta}^{\dagger}= & F_{\boldsymbol{q} \beta}^{\dagger} \mathcal{E}_{\boldsymbol{q} \beta}+i \sum_{\beta^{\prime}} F_{\boldsymbol{q} \beta^{\prime}}^{\dagger} \mathcal{I}_{\boldsymbol{q} \beta^{\prime} \beta} \\
& +\sum_{\beta^{\prime}} \mathcal{M}_{-\boldsymbol{q} \beta \beta^{\prime}}^{\prime \prime} F_{-\boldsymbol{q} \beta^{\prime}}
\end{aligned}
$$

where $F_{\boldsymbol{q}}$ is Hermitian conjugate of $F_{\boldsymbol{q}}^{\dagger}$,

$$
\mathcal{M}_{-\boldsymbol{q} \beta \beta^{\prime}}^{\prime \prime}=\left(\sum_{\alpha} M_{-\boldsymbol{q} \alpha}^{\prime \prime} V_{\boldsymbol{q} \alpha \beta}^{R}\right) V_{-\boldsymbol{q} \alpha_{2} \beta^{\prime}}^{L},
$$

and

$$
\mathcal{I}_{\boldsymbol{q}}=I_{\boldsymbol{q}}+\frac{\gamma_{\boldsymbol{q}}^{(0)}}{2}
$$

Here, $I_{\boldsymbol{q}}$ is a nonadiabatic interaction given by

$$
I_{\boldsymbol{q} \beta^{\prime} \beta}=\sum_{\alpha \alpha^{\prime} \alpha^{\prime \prime}} \frac{d\left(V_{\boldsymbol{q} \beta^{\prime} \alpha^{\prime}}^{L \dagger} U_{\boldsymbol{q} \alpha^{\prime} \alpha^{\prime \prime}}^{L \dagger}\right)}{d t}\left(U_{\boldsymbol{q} \alpha^{\prime \prime} \alpha}^{R} V_{\boldsymbol{q} \alpha \beta}^{R}\right)
$$

with $I_{\boldsymbol{q}} \neq-I_{\boldsymbol{q}}^{\dagger}$, and a phenomenological damping factor $\gamma_{\boldsymbol{q} \beta^{\prime} \beta}^{(0)}$ pertinent to $F_{\boldsymbol{q} \beta}^{\dagger}$ is given by

$$
\frac{\gamma_{\boldsymbol{q} \beta^{\prime} \beta}^{(0)}}{2}=\sum_{\alpha \alpha^{\prime}} V_{\boldsymbol{q} \beta^{\prime} \alpha^{\prime}}^{L \dagger} \frac{\gamma_{\boldsymbol{q} \alpha^{\prime} \alpha}^{(B)}}{2} V_{\boldsymbol{q} \alpha \beta}^{R} .
$$

Hereafter, the operator $F_{q \beta}^{\dagger}(t)$ thus introduced is termed as a creation operator of $\mathrm{PQ}$, and then the corresponding annihilation operator is $F_{q \beta}(t)$; these are not bosonic operators. The bosonization scheme for the PQ operators is similar to that for the quasiboson operators [104], where the PQ ground state is given by the direct product of the ground states of quasiboson and LO phonon, and $\mathcal{E}_{\boldsymbol{q} \beta}(t)$ is the single-PQ adiabatic energy at time $t$ with mode $\boldsymbol{q} \beta$. In terms of $F_{\boldsymbol{q}}$ and $F_{\boldsymbol{q}}^{\dagger}$, the associated retarded Green function is given by [105]

$$
G_{q \beta \beta^{\prime}}^{R}\left(t, t^{\prime}\right)=-i \theta\left(t-t^{\prime}\right)\left\langle\left[F_{\boldsymbol{q} \beta}(t), F_{\boldsymbol{q} \beta^{\prime}}^{\dagger}\left(t^{\prime}\right)\right]\right\rangle .
$$

\section{Approximations employed}

The major approximation made thus far is the factorization approximation implemented in Eqs. (12) and (32) in addition to the rotational-wave approximation implemented in Eq. (20), aside from other unimportant assumptions. Here, further approximations are made for actual calculations as follows. (i) Time integration for solving Eq. (42) begins at $t=t_{D}$ with 
$t_{D}=\tau_{L} / 2, \tau_{L}$ being defined in Eq. (19), and contributions from the temporal region $t \leqslant t_{D}$ are neglected. (ii) The relation of $\left\langle\left[B_{\boldsymbol{q} \alpha}, B_{\boldsymbol{q}^{\prime} \alpha^{\prime}}^{\dagger}\right]\right\rangle=\delta_{\boldsymbol{q} \boldsymbol{q}^{\prime}} \delta_{\alpha \alpha^{\prime}}$ is assumed, so that Eq. (34) is given by $M_{q \alpha \alpha^{\prime}}^{\prime}=M_{q \alpha \alpha^{\prime}}$. (iii) Effects of $M_{-q \alpha \alpha^{\prime}}^{\prime \prime}$ of Eq. (33) on Eqs. (35) and (42) are neglected. (iv) Equation (46) is given by $\gamma_{\boldsymbol{q} \beta^{\prime} \beta}^{(0)}(t)=\delta_{\beta^{\prime} \beta} \gamma_{\boldsymbol{q}} \theta\left(t+t_{D}\right)$ with $\gamma_{\boldsymbol{q}}$ a positive and real constant.

The approximation (i) is valid here since $\tau_{L}$ is much shorter than the carrier-density relaxation time of the order of $100 \mathrm{fs}$. Thus, in the temporal region $t>t_{D}$, due to the rotational-wave approximation, the adiabatic picture that forms the basis of the PQ picture is justified, and the effects of nonadiabatic interactions $I_{\boldsymbol{q} \beta^{\prime} \beta}$ are neglected. As regards the approximation (ii), the criterion of validity of it is discussed in Ref. [102]. This criterion is ensured in the same temporal region as mentioned above, where the Coulomb correction to the Rabi frequency $\sum_{\boldsymbol{q}} V_{\boldsymbol{q}}^{(C)} \bar{\rho}_{b b^{\prime} \boldsymbol{k}+\boldsymbol{q}}$ given in Eq. (21) becomes much smaller than $\omega_{q}^{(\mathrm{LO})}$. This approximation brings in the train the demand of employing the limited set $\{\alpha\}$ with $\mathcal{E}_{\boldsymbol{q} \alpha}$ being real and positive out of all the solutions of Eqs. (22) and (23). Owing to this, the matrix $h_{\boldsymbol{q}}$ of Eq. (38) becomes Hermitian, and $V_{\boldsymbol{q} \beta}^{R}$ and $V_{\boldsymbol{q} \beta}^{L}$ are identical. Thus, a set of the vectors are readily obtained [60]. The approximation (iii) is due to the present quasibosonization scheme in which the contribution of two-quasiboson states is discarded, as stated in Ref. [103]. Actually, $M_{-q \alpha \alpha^{\prime}}^{\prime \prime}$ is attributed to an interquasiboson interaction accompanying momentum transfer from $-\boldsymbol{q}$ to $\boldsymbol{q}$. The approximation (iv) is derived from the supposition that $T_{q k b b^{\prime}}$ given in Eq. (10) is independent of $\boldsymbol{k}, b$, and $b^{\prime}$, and is commonly labeled as $T_{\boldsymbol{q} 12}: \gamma_{\boldsymbol{q}}=2 / T_{\boldsymbol{q} 12}$.

\section{B. Physical quantities for transient FR}

\section{Longitudinal susceptibility}

According to the linear response theory, an induced electron density $n_{\boldsymbol{q}}^{(i n d)}(t)$ introduced by a weak external potential $f_{\boldsymbol{q}}(t)$ is provided by $[105,106]$

$$
n_{\boldsymbol{q}}^{(\mathrm{ind})}(t)=\frac{1}{4 \pi V} \int_{-\infty}^{t} d t^{\prime} \chi_{\boldsymbol{q}}^{(t)}\left(t, t^{\prime}\right) f_{\boldsymbol{q}}\left(t^{\prime}\right)
$$

Here, $\chi_{\boldsymbol{q}}^{(t)}\left(t, t^{\prime}\right)$ represents the retarded longitudinal susceptibility in the transient and nonequilibrium system of concern. This depends on passage of $t$ as well as the relative time $\tau=t-t^{\prime}$ in the present system, differing from equilibrium systems depending solely on $\tau$ due to temporal translational invariance being conserved. $\chi_{q}^{(t)}\left(t, t^{\prime}\right)$ is provided by the sum of a retarded susceptibility due to the electron-induced interaction $\chi_{q}\left(t, t^{\prime}\right)$ and that of an LO-phonon-induced interaction $\chi_{q}^{\prime}\left(t, t^{\prime}\right)$, namely [105],

$$
\chi_{\boldsymbol{q}}^{(t)}\left(t, t^{\prime}\right)=\chi_{\boldsymbol{q}}\left(t, t^{\prime}\right)+\chi_{\boldsymbol{q}}^{\prime}\left(t, t^{\prime}\right)
$$

In the case that $f_{q}\left(t^{\prime}\right)$ is given by a delta pulse as $f_{q}\left(t^{\prime}\right)=$ $f_{q 0} \delta\left(t^{\prime}-t_{p}\right)$, Eq. (48) becomes

$$
n_{\boldsymbol{q}}^{(\mathrm{ind})}(t)=\frac{f_{\boldsymbol{q} 0}}{4 \pi V} \chi_{\boldsymbol{q}}^{(t)}\left(t, t_{p}\right)
$$

where $f_{\boldsymbol{q} 0}$ is independent of $t^{\prime}$, and $t_{p}$ represents the time at which $f_{\boldsymbol{q}}\left(t^{\prime}\right)$ probes transient dynamics of concern. Thus, it is seen that $\chi_{\boldsymbol{q}}^{(t)}\left(t, t_{p}\right)$ reveals the way of alteration in the induced electron density after $t_{p}$.
The inverse dielectric function $\epsilon_{q}^{-1}\left(t_{p}+\tau, t_{p}\right)$ is given as

$$
\epsilon_{\boldsymbol{q}}^{-1}\left(t_{p}+\tau, t_{p}\right)=\epsilon_{\infty}^{-1}\left[\delta(\tau)+\frac{V}{4 \pi} v_{\boldsymbol{q}}^{(C)} \chi_{\boldsymbol{q}}^{(t)}\left(t_{p}+\tau, t_{p}\right) \theta(\tau)\right],
$$

where $v_{\boldsymbol{q}}^{(C)}=\epsilon_{\infty} V_{\boldsymbol{q}}^{(C)}$ with $\epsilon_{\infty}$ as a background dielectric constant given in Eq. (3). The Fourier transform of the dielectric function $\epsilon_{\boldsymbol{q}}\left(t_{p}+\tau, t_{p}\right)$,

$$
\tilde{\epsilon}_{\boldsymbol{q}}\left(t_{p} ; \omega\right)=\int_{0}^{\infty} d \tau e^{-i \omega \tau} \epsilon_{\boldsymbol{q}}\left(t_{p}+\tau, t_{p}\right),
$$

is readily obtained by employing the relation $\int_{-\infty}^{\infty} d t^{\prime \prime} \epsilon_{q}^{-1}\left(t_{p}+\right.$ $\left.\tau, t^{\prime \prime}\right) \epsilon_{q}\left(t^{\prime \prime}, t_{p}\right)=\delta(\tau)$. Therefore, a transient absorption coefficient $\alpha_{\boldsymbol{q}}\left(t_{p} ; \omega\right)$ at time $t_{p}$ is given by the following expression:

$$
\alpha_{q}\left(t_{p} ; \omega\right)=\frac{\omega}{n\left(t_{p} ; \omega\right) C} A_{q}\left(t_{p} ; \omega\right),
$$

where

$$
A_{q}\left(t_{p} ; \omega\right)=\operatorname{Im} \tilde{\epsilon}_{q}\left(t_{p} ; \omega\right) .
$$

Here, $n\left(t_{p} ; \omega\right)$ represents the index of refraction, which is approximately given by $n\left(t_{p} ; \omega\right) \approx \sqrt{\epsilon_{\infty}}$, and $C$ represents the speed of light. There are two remarks regarding $A_{q}\left(t_{p} ; \omega\right)$. One is that this is nonlinear in the pump field of Eq. (18). The other is that contrary to convention, the sign of $\omega$ is defined in Eq. (52) such that transient photoemission spectra $A_{q}\left(t_{p} ; \omega\right)<$ 0 peak at positive $\omega$, while transient photoabsorption spectra $A_{q}\left(t_{p} ; \omega\right)>0$ peak at negative $\omega$. The transient induced photoemission spectra are defined as

$$
\bar{A}_{\boldsymbol{q}}\left(t_{p} ; \omega\right)=-A_{\boldsymbol{q}}\left(t_{p} ; \omega\right) .
$$

Below, explicit expressions of $\chi_{q}\left(t, t^{\prime}\right)$ and $\chi_{q}^{\prime}\left(t, t^{\prime}\right)$ are derived based on the PQ model. One begins with $\chi_{q}\left(t, t^{\prime}\right)$ associated with a retarded density-density correlation function of electron defined as [105,106]

$$
i D_{q}^{R}\left(t, t^{\prime}\right)=\theta\left(t-t^{\prime}\right)\left\langle\left[\hat{n}_{\boldsymbol{q}}(t), \hat{n}_{-\boldsymbol{q}}\left(t^{\prime}\right)\right]\right\rangle,
$$

where a density operator is defined as

$$
\hat{n}_{\boldsymbol{q}}(t)=\frac{\hat{\rho}_{\boldsymbol{q}}(t)}{V}=\frac{1}{V} \sum_{b \boldsymbol{k}} a_{b \boldsymbol{k}+\boldsymbol{q}}^{\dagger} a_{b \boldsymbol{k}}
$$

in terms of Eq. (4). It is remarked that

$$
\hat{n}_{-\boldsymbol{q}}(t)=\hat{n}_{\boldsymbol{q}}^{\dagger}(t)
$$

and, hence,

$$
D_{q}^{R^{\dagger}}\left(t, t^{\prime}\right)=D_{-q}^{R}\left(t, t^{\prime}\right) .
$$

Employing the relations of Eq. (25) brings Eq. (57) to

$$
\hat{n}_{\boldsymbol{q}}(t)=\frac{1}{V} \sum_{\alpha} B_{\boldsymbol{q} \alpha}^{\dagger} N_{\boldsymbol{q} \alpha}^{L}
$$

where $N_{\boldsymbol{q} \alpha}^{L}=\sum_{\boldsymbol{k} b} U_{\boldsymbol{q} \alpha}^{L \dagger}(\boldsymbol{k} b b)$ that is equivalent to a normalization constant of the left vector $U_{\boldsymbol{q} \alpha}^{L \dagger}$ [102]. According to this expression, it is readily seen that $B_{q \alpha}^{\dagger}$ represents a fraction 
of electron density at the state of $\alpha$ weighted with $N_{q \alpha}^{L} / V$. Following Eq. (41), Eq. (60) is recast into

$$
\hat{n}_{\boldsymbol{q}}(t)=\frac{1}{V} \sum_{\beta \alpha} F_{\boldsymbol{q} \beta}^{\dagger} V_{\boldsymbol{q} \beta \alpha}^{L \dagger} N_{\boldsymbol{q} \alpha}^{L} .
$$

Since $\chi_{\boldsymbol{q}}\left(t, t^{\prime}\right)$ is expressed in terms of $D_{q}^{R}\left(t, t^{\prime}\right)$ as

$$
\chi_{q}\left(t, t^{\prime}\right) \equiv 4 \pi V D_{q}^{R}\left(t, t^{\prime}\right)=\chi_{-q}^{*}\left(t, t^{\prime}\right),
$$

this is given by

$$
\begin{aligned}
\chi_{\boldsymbol{q}}^{*}\left(t, t^{\prime}\right)= & \frac{4 \pi}{V} \sum_{\alpha \alpha^{\prime} \beta \beta^{\prime}} N_{\boldsymbol{q} \alpha}^{L *}(t) V_{\boldsymbol{q} \alpha \beta}^{L}(t) G_{\boldsymbol{q} \beta \beta^{\prime}}^{R}\left(t, t^{\prime}\right) \\
& \times V_{\boldsymbol{q} \beta^{\prime} \alpha^{\prime}}^{L \dagger}\left(t^{\prime}\right) N_{\boldsymbol{q} \alpha^{\prime}}^{L}\left(t^{\prime}\right) .
\end{aligned}
$$

On the other hand, $\chi_{\boldsymbol{q}}^{\prime}\left(t, t^{\prime}\right)$ in Eq. (49) is expressed as

$$
\chi_{\boldsymbol{q}}^{\prime}\left(t, t^{\prime}\right)=\frac{4 \pi}{V}\left|g_{\boldsymbol{q}}^{\prime}\right|^{2} D_{\boldsymbol{q}}^{\prime R}\left(t, t^{\prime}\right),
$$

where

$$
\left|g_{\boldsymbol{q}}^{\prime}\right|^{2}=\left|\frac{g_{\boldsymbol{q}}^{0}}{v_{\boldsymbol{q}}^{(C)}}\right|^{2}
$$

with $g_{\boldsymbol{q}}^{0}=\left(g_{c \boldsymbol{q}}+g_{v \boldsymbol{q}}\right) / 2$. Further, $D_{q}^{\prime R}\left(t, t^{\prime}\right)$ is a retarded phonon Green function given by [105]

$$
D_{q}^{\prime R}\left(t, t^{\prime}\right) \equiv \bar{D}_{q}^{\prime R}\left(t, t^{\prime}\right)+\left[\bar{D}_{-q}^{\prime R}\left(t, t^{\prime}\right)\right]^{*},
$$

where

$$
\bar{D}_{\boldsymbol{q}}^{\prime R}\left(t, t^{\prime}\right)=-i\left\langle\left[c_{\boldsymbol{q}}(t), c_{\boldsymbol{q}}^{\dagger}\left(t^{\prime}\right)\right]\right\rangle \theta\left(t-t^{\prime}\right),
$$

and employing Eq. (41), this is cast into

$$
\bar{D}_{q}^{\prime R}\left(t, t^{\prime}\right)=\sum_{\beta \beta^{\prime}} V_{q \alpha_{2} \beta}^{L}(t) G_{q \beta \beta^{\prime}}^{R}\left(t, t^{\prime}\right) V_{\boldsymbol{q} \beta^{\prime} \alpha_{2}}^{L \dagger}\left(t^{\prime}\right) .
$$

Lastly, it is mentioned that the normalization constant $N_{\boldsymbol{q} \alpha}^{R}(t)$ of the $\alpha$ th solution $U_{\boldsymbol{q}_{\alpha}}^{R}(t)$ of Eq. (23) is undetermined up to an overall phase factor $e^{i \eta_{q \alpha}(t)}$, where $\eta_{q \alpha}(t)$ is an arbitrary real function of $t$. Such arbitrariness brings the quasiboson operators $B_{q_{\alpha}}^{\dagger}(t)$ and $B_{q \alpha}(t)$ to be transformed such that $B_{q \alpha}^{\dagger}(t) \rightarrow B_{q \alpha}^{\dagger}(t) e^{i \eta_{q \alpha}(t)}$ and $B_{q \alpha}(t) \rightarrow B_{q \alpha}(t) e^{-i \eta_{q \alpha}(t)}$, respectively. It is certain that $\chi_{q}^{(t)}\left(t, t^{\prime}\right)$ of Eq. (49) is invariant with respect to these phase transformations and independent of any choice of $\eta_{\boldsymbol{q} \alpha}(t)$. This invariance is proved in Ref. [102].

\section{Power spectra of phonon displacement function}

An expectation value of $\left[c_{\boldsymbol{q}}(t)+c_{-q}^{\dagger}(t)\right] / 2$ with respect to the ground state, namely,

$$
Q_{q}(t)=\frac{1}{2}\left\langle c_{q}(t)+c_{-q}^{\dagger}(t)\right\rangle,
$$

is examined, where the phonon operators $c_{q}$ and $c_{-q}^{\dagger}$ are extracted from the PQ operator by means of the projection given by Eq. (41). This is regarded as a classical phonon displacement function in the case that this expectation value is taken with respect to a coherent state of phonon. In addition, this is also considered as transition probability of Raman scattering process [57]. As a consequence of the

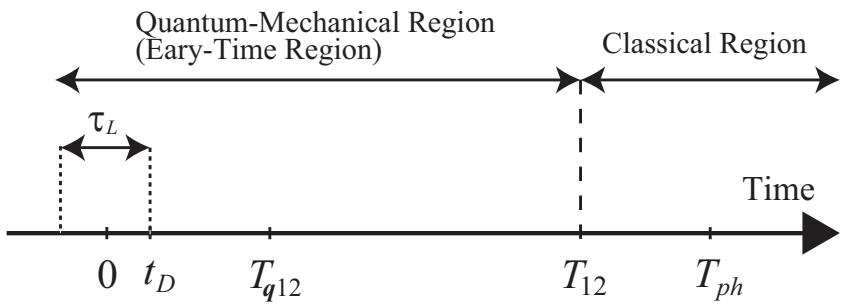

FIG. 1. Schematic allocation of various time constants employed in actual calculations. For more detail, consult the text.

Wiener-Khintchine's theorem [99], the Fourier transform of the two-time correlation

$$
C_{q}(t)=\int_{-\infty}^{\infty} Q_{q}\left(t+t^{\prime}\right) Q_{q}\left(t^{\prime}\right) d t^{\prime}
$$

provides the spectral function

$$
S_{\boldsymbol{q}}(\omega)=\int_{-\infty}^{\infty} e^{-i \omega t} C_{\boldsymbol{q}}(t) d t
$$

Employing the Fourier transform of $Q_{q}(t)$,

$$
\tilde{Q}_{q}(\omega)=\int_{0}^{\infty} e^{-i \omega t} Q_{q}(t) d t,
$$

it is readily seen that $S_{q}(\omega)$ is proportional to the power spectra, that is,

$$
S_{q}(\omega) \propto\left|\tilde{Q}_{q}(\omega)\right|^{2} .
$$

\section{Setup parameters for actual calculations}

Materials parameters of both undoped Si and undoped GaAs and laser parameters employed for actual calculations are listed in Ref. [102]. Opaque interband transitions accompanying real excited carriers are exclusively considered. The crystals are assumed to be cubic. The concerned material of undoped $\mathrm{Si} / \mathrm{GaAs}$ is referred to just as $\mathrm{Si} / \mathrm{GaAs}$ hereafter unless otherwise stated. Here, the single-particle density matrices $\rho_{b b^{\prime} \boldsymbol{k}}(t)$ are evaluated in advance by solving optical Bloch equations within the two-band model composed of $c$ and $v$ bands. Carrier-density relaxation time, labeled as $T_{1}$, and dephasing time, labeled as $T_{2}$, are set identical each other: $T_{12} \equiv T_{1}=T_{2} . T_{12}$ and $T_{q 12}$ (defined in Sec. II A 4) represent phenomenological damping time constants of induced carrier density with isotropic and anisotropic momentum distributions, respectively. The temporal region $t<T_{12}$ corresponds to the early-time region during which a great number of carriers still stay in excited states (see Sec. I), while the temporal region $t \gtrsim T_{12}$ is termed as the classical region; the former is also termed as the quantum-mechanical region since the quantum processes govern the $\mathrm{CP}$ dynamics.

Figure 1 shows schematic allocation of the various time constants mentioned above, namely, $t_{D}, T_{q 12}, T_{12}$, and $T_{p h}$; the last one represents a phenomenological damping constant of LO phonon due to lattice anharmonicity.

Time scales for different stages of relaxation phenomena in photoexcited experiments are summarized in Ref. [3]. For the present calculations, $t_{D}, T_{q 12}, T_{12}$, and $T_{p h}$ are set equal to 7.5, 20, 90, and $5000 \mathrm{fs,} \mathrm{respectively.} \mathrm{Such} \mathrm{an} \mathrm{allocation} \mathrm{of} \mathrm{these}$ time constants shown in Fig. 1 is a necessary condition for the 
manifestation of the FR of concern in $\mathrm{Si}$, as discussed in more detail in Sec. III B.

As regards experimental estimates of the time constants for $\mathrm{Si}, t_{D}, T_{q 12}, T_{12}$, and $T_{p h}$ extracted from the $\mathrm{CP}$ measurements in Ref. [13] are 5, 16, 100, and $1300 \mathrm{fs}$, respectively, at average excited electron density $\bar{N}_{e x}=4 \times 10^{19} \mathrm{~cm}^{-3}$; further, $T_{p h}$ obtained from the Raman scattering in $\mathrm{Si}$ is approximately $2500 \mathrm{fs}$ at low temperature [76], $1330 \mathrm{fs}$ at $300 \mathrm{~K}$ [76], and 1970 fs at $300 \mathrm{~K}$ [87]. In Ref. [12], $T_{q 12}$ is estimated as $32 \pm 5 \mathrm{fs}$ at $\bar{N}_{e x}=(5.5 \pm 0.3) \times 10^{18} \mathrm{~cm}^{-3}$ in the pumpprobe reflectivity study of carrier dynamics. Incidentally, $T_{12}$ for GaAs extracted from numerical calculations in Ref. [107] is 50 fs at $\bar{N}_{e x}=3 \times 10^{18} \mathrm{~cm}^{-3}$.

\section{RESULTS AND DISCUSSION}

\section{A. Adiabatic energy configuration}

Figure 2 shows adiabatic energy curves $\mathcal{E}_{q \alpha}(t)$ of quasiboson in $\mathrm{Si}$ as a function of $t$ in the small- $\boldsymbol{q}$ limit concerned here. The energy indicated by a green solid line is $\mathcal{E}_{\boldsymbol{q} \alpha_{1}}(t)$ attributed to a plasmonlike mode. Indeed, this looks almost proportional to the plasmon energy $\omega_{p l}$ indicated by a broken line in this figure with $\omega_{p l}^{2}=4 \pi N_{e x}(t) / \epsilon_{\infty} m_{c v}$, where $N_{e x}(t)=(1 / V) \sum_{k} \bar{\rho}_{c c k}$ is an excited electron density, and $m_{c v}$ is reduced mass of electrons in a joint band composed of $c$ and $v$ bands under an effective mass approximation; the maximum of $N_{e x}(t)$ is $6.31 \times 10^{17} \mathrm{~cm}^{3}$. In fact, $\mathcal{E}_{q \alpha_{1}}(t)$ depends on interband density matrices of $\bar{\rho}_{c v k}(t)$ and $\bar{\rho}_{v c k}(t)$, and Rabi frequencies of $\bar{\Omega}_{c v k}^{(R)}(t)$ and $\bar{\Omega}_{v c k}^{(R)}(t)$ in a complicated manner, as well as on the intraband density matrices of $\bar{\rho}_{c c k}(t)$ and $\bar{\rho}_{v v k}(t)$ [102]. Thus, the difference of the functional shapes between $\mathcal{E}_{\boldsymbol{q} \alpha_{1}}(t)$ and $\omega_{p l}$ is definitely attributed to the transient effect of the interband density matrices and the Rabi frequencies. On the occasion that such effects are suppressed in the time region of $t>t_{D}, \mathcal{E}_{q \alpha_{1}}(t)$ becomes identical to $\omega_{p l}$,

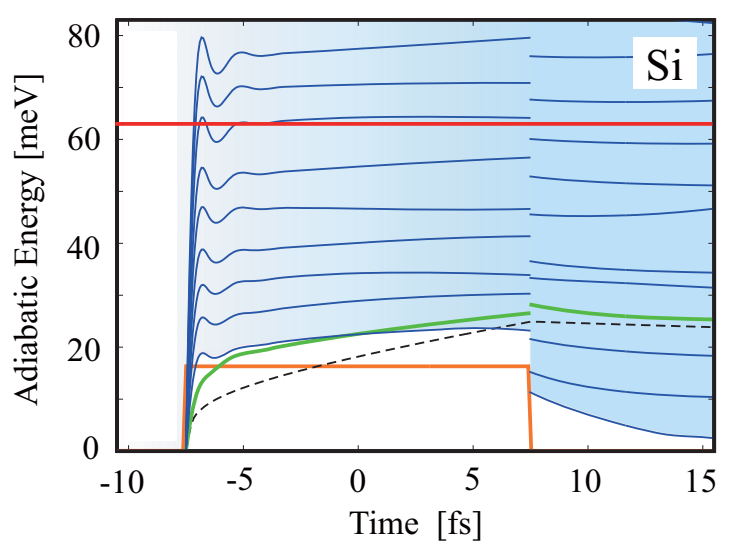

FIG. 2. Adiabatic energy curves of $\mathrm{Si}$ (in the unit of $\mathrm{meV}$ ) as a function of time $t$ (in the unit of fs). $\mathcal{E}_{\boldsymbol{q} \alpha_{1}}(t),\left\{\mathcal{E}_{\boldsymbol{q} \beta}(t)\right\}, \omega_{q}^{(\mathrm{LO})}, \Omega_{0 c v}$, and $\omega_{p l}$ are represented by a green solid line, blue solid lines, a red solid line, an orange solid line, and a broken line, respectively. The gradation of blue color shows schematic change of $N_{e x}(t)$ in $t$, where the lowest limit of this gradation represents the threshold energy of $\left\{\mathcal{E}_{q \beta}(t)\right\}$. For more detail, consult the text. aside from a renormalization effect due to $V_{q}^{(C)}$ on the Rabi frequencies.

Discretized adiabatic-energy levels indicated by blue solid lines show a bundle of electron-hole continua $\left\{\mathcal{E}_{\boldsymbol{q} \beta}(t)\right\}$, the lowest energy of which delineates a threshold of these contributions. This threshold is approximately given by $\left|2 \bar{\Omega}_{c v k}^{(R)}(t)\right|$ [102]; just for the purpose of comparison, $\Omega_{0 c v}$, corresponding to the first term of Eq. (21), is represented by an orange solid line. The energy discretization for $\left\{\mathcal{E}_{\boldsymbol{q} \beta}(t)\right\}$ results from the incorporation of the finite number of sites in the calculations: $V=N_{s} d^{3}$ and $N_{s}=70^{3}$ with $d$ as a lattice constant. It is noted that the formation of the continua $\left\{\mathcal{E}_{\boldsymbol{q} \beta}(t)\right\}$ exclusively arises from interband transitions; in fact, the effect of intraband transitions vanishes in the small- $\boldsymbol{q}$ limit. The gradation of blue color shows schematic change of $N_{e x}(t)$ in $t$. The LO-phonon energy $\omega_{q}^{(\mathrm{LO})}=63 \mathrm{meV}$ is also represented by a red solid line. Adiabatic energy curves of $\mathcal{E}_{q \alpha}(t)$ of GaAs as a function of $t$ show the similar behavior as those of $\mathrm{Si}$, though not depicted here.

As is seen in Fig. 2, the LO-phonon mode $\alpha_{2}$ is embedded to the electron-hole continuum state $\beta$ right after the onset of the laser irradiation. Evidently, this effect lasts even after the completion of laser irradiation: $t>t_{D}$. It is likely that $M_{q \beta}$ given by Eq. (31) causes a coupling of the LO-phonon state with the quasiboson continuum state $\beta$ in a resonant manner, namely, at $\mathcal{E}_{q \beta} \approx \omega_{q}^{(\mathrm{LO})}$, to generate FR in the occasion that the excited electron density is high enough: $M_{q \beta}$ depends on the excited electron density. Such an energy configuration is a substantial condition to be fulfilled for the manifestation of the FR in addition to the above-mentioned condition regarding the allocation of time constants. The plasmonlike mode $\alpha_{1}$ tends to dive into the continua in $t>t_{D}$. However, no FR attributed to this mode is expected to appear since the coupling of it with the continuum state $\beta$ is given by the second-order interaction of the form $M_{q \beta} M_{q \alpha_{1}}^{*}$ mediated by the LO phonon, and this is considered negligibly small, as shown later in Sec. III B.

Figure 2 also shows that the adiabatic energy curves in $t>t_{D}$ are such slowly time-varying functions that the $\mathrm{PQ}$ model based on the adiabatic picture is ensured; see also the approximation (i) in Sec. II A 4 . The discontinuity at $t=$ $\pm \tau_{L} / 2$ is due to the pulse shape of Eq. (19).

\section{B. Transient induced photoemission spectra}

Transient induced photoemission spectra $\bar{A}_{q}\left(t_{p}, \omega\right)$ of Eq. (55) show the change of excited electronic structure formed by a nonlinear optical process due to the pump field at probe time $t_{p}$. Thus, this is a crucial observable to understand manifestation of transient and nonlinear FR accompanied by CP generation. Here, $\bar{A}_{q}\left(t_{p} ; \omega\right)$ 's of Si and GaAs as a function of frequency $\omega$ are taken into account. As seen from Eq. (49), the two interactions, the dynamically screened Coulomb interaction induced by electron and the LOphonon-induced interaction, contribute to the total retarded longitudinal susceptibility, that is,

$$
\tilde{\chi}_{\boldsymbol{q}}^{(t)}\left(t_{p} ; \omega\right)=\tilde{\chi}_{\boldsymbol{q}}\left(t_{p} ; \omega\right)+\tilde{\chi}_{\boldsymbol{q}}^{\prime}\left(t_{p} ; \omega\right),
$$

where $\tilde{\chi}_{q}^{(t)}\left(t_{p} ; \omega\right), \tilde{\chi}_{q}\left(t_{p} ; \omega\right)$, and $\tilde{\chi}_{q}^{\prime}\left(t_{p} ; \omega\right)$ are Fourier transforms of $\chi_{q}^{(t)}\left(t_{p}+\tau, t_{p}\right), \chi_{\boldsymbol{q}}\left(t_{p}+\tau, t_{p}\right)$, and $\chi_{q}^{\prime}\left(t_{p}+\tau, t_{p}\right)$ with 
respect to $\tau$ into the $\omega$ domain, respectively, similarly to Eq. (52). In the small- $\boldsymbol{q}$ limit, $\tilde{\chi}_{\boldsymbol{q}}\left(t_{p} ; \omega\right)$ is in proportion to $|\boldsymbol{q}|^{2}$. On the other hand, because of Eq. (65), $\tilde{\chi}_{\boldsymbol{q}}^{\prime}\left(t_{p} ; \omega\right)$ is in proportion to $|\boldsymbol{q}|^{2}$ for the Fröhlich interaction and to $|\boldsymbol{q}|^{4}$ for the deformation potential interaction. This difference arises from the fact that the former interaction is of long range, and the latter one is of short range, which reflects on $\bar{A}_{q}\left(t_{p}, \omega\right)$ through Eq. (51), as it should be; in a nonpolar crystal such as $\mathrm{Si}$, due to the presence of spatial inversion symmetry, lattice absorption vanishes in the limit of a dipole transition with transferred momentum $\boldsymbol{q}$ being zero [108].

Figures 3 and 4 show $\bar{A}_{q}\left(t_{p} ; \omega\right)$ of $\mathrm{Si}$ and GaAs, respectively, which are indicated by red lines at $t_{p}=15,65$, and $100 \mathrm{fs}$. Here, the separate contributions from $\tilde{\chi}_{\boldsymbol{q}}\left(t_{p} ; \omega\right)$ and $\tilde{\chi}_{\boldsymbol{q}}^{\prime}\left(t_{p} ; \omega\right)$ are shown by blue and green lines, respectively. The former and the latter are mostly governed by the plasmonlike mode $\alpha_{1}$ and the LO-phonon mode $\alpha_{2}$, respectively; $\tilde{\chi}_{q}\left(t_{p} ; \omega\right)$ is due to electronic excitation mediated by optical interband transitions. It is understood that $\bar{A}_{\boldsymbol{q}}\left(t_{p} ; \omega\right)$ is reckoned from structureless background spectra ascribable to electron-hole continuum states $\beta$ that are almost constant in the $\omega$ region concerned. In both figures, it is seen that just $\tilde{\chi}_{q}^{\prime}\left(t_{p} ; \omega\right)$ contributes to the formation of spectral peaks, and $\tilde{\chi}_{q}^{\prime}\left(t_{p} ; \omega\right)$ becomes dominant over $\tilde{\chi}_{q}\left(t_{p} ; \omega\right)$ in the region $t_{p} \gtrsim T_{12}$. In passing, the widths of the spectral peaks are determined by $2 / T_{p h}=0.27 \mathrm{meV}$ rather than natural spectral width.

Figure 3(a) shows $\bar{A}_{q}\left(t_{p} ; \omega\right)$ of $\mathrm{Si}$ at $t_{p}=15 \mathrm{fs}$, where the spectra are governed by the contribution from $\tilde{\chi}_{q}\left(t_{p} ; \omega\right)$ due to the formation of the plasmonlike $\alpha_{1}$ mode, namely, $\tilde{\chi}_{q}^{(t)}\left(t_{p} ; \omega\right) \approx \tilde{\chi}_{q}\left(t_{p} ; \omega\right)$, and the continuum spectra decrease monotonically in $\omega$. On the contrary, the contribution from $\tilde{\chi}_{q}^{\prime}\left(t_{p} ; \omega\right)$ is negligibly small due to the proportion of it to $|\boldsymbol{q}|^{4}$. As is shown in Fig. 3(b), at $t_{p}=65$ fs, the contributions from $\tilde{\chi}_{q}\left(t_{p} ; \omega\right)$ are damped to be comparable to those from $\tilde{\chi}_{q}^{\prime}\left(t_{p} ; \omega\right)$. It should be noted that asymmetric spectra are manifested with a dip followed by a peak reminiscent of FR. The resulting spectral profile is in sharp contrast with a symmetric Lorentzian profile shown in Fig. 3(c) at $t_{p}=$ $100 \mathrm{fs}$, at which the spectra are determined by $\tilde{\chi}_{q}^{\prime}\left(t_{p} ; \omega\right)$ and $\tilde{\chi}_{q}^{(t)}\left(t_{p} ; \omega\right) \approx \tilde{\chi}_{q}^{\prime}\left(t_{p} ; \omega\right)$.

As regards $\bar{A}_{q}\left(t_{p} ; \omega\right)$ of GaAs, it is shown in Fig. 4(a) that at $t_{p}=15 \mathrm{fs}$, a discernible peak due to the $\alpha_{2}$ mode is superimposed with a continuum background. This background is composed of the contributions of $\tilde{\chi}_{\boldsymbol{q}}\left(t_{p} ; \omega\right)$ and $\tilde{\chi}_{q}^{\prime}\left(t_{p} ; \omega\right)$ with comparable order since both are in proportion to $|\boldsymbol{q}|^{2}$. The spectra at $t_{p}=65$ fs shown in Fig. 4(b) are dominated by $\tilde{\chi}_{q}^{\prime}\left(t_{p} ; \omega\right)$. The spectral profile differs a lot from that shown in Fig. 3(b) of $\mathrm{Si}$ in that the former spectra are of symmetric shape. The spectra at $t_{p}=100 \mathrm{fs}$ in Fig. 4(c) are similar to those in Fig. 3(c).

The manifestation of the transient $\mathrm{FR}$ in $\mathrm{Si}$ shown in Fig. 3(b) can be tracked to its origin by inspecting the details of numerical calculations and by reducing Eq. (68) to an approximated analytic expression, though not shown here. Due to these procedures, it is identified that the principal difference between $\mathrm{Si}$ and GaAs observed here arises just from the effective coupling $M_{q \beta}$ between quasiboson and LO phonon given by Eq. (31) aside from less significant difference in other material parameters. The primitive coupling
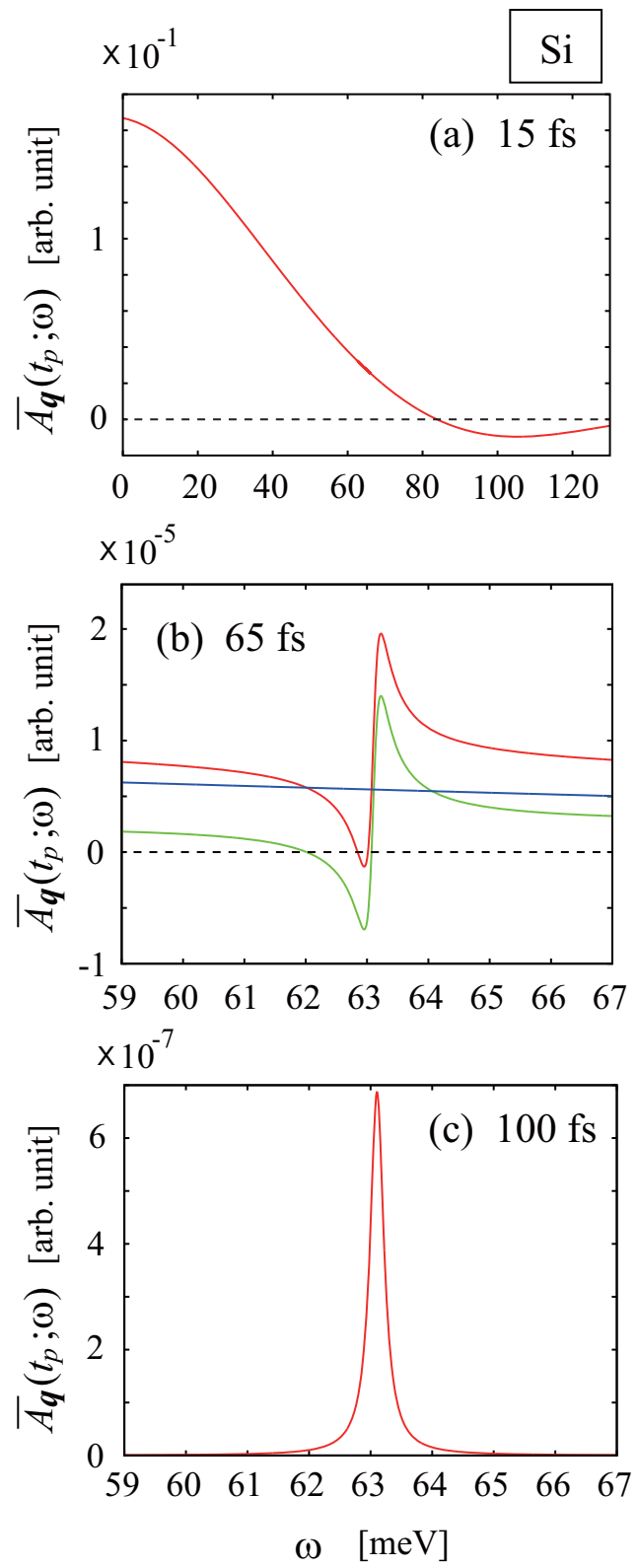

FIG. 3. Transient photoemission spectra $\bar{A}_{q}\left(t_{p}, \omega\right)$ of $\mathrm{Si}$ (red line) as a function of frequency $\omega$ (in the unit of $\mathrm{meV}$ ) at probe time $t_{p}$ of (a) $15 \mathrm{fs}$, (b) $65 \mathrm{fs}$, and (c) $100 \mathrm{fs}$. Separate contributions to the spectra from $\tilde{\chi}_{q}\left(t_{p} ; \omega\right)$ and $\tilde{\chi}_{q}^{\prime}\left(t_{p} ; \omega\right)$ are also shown by blue and green lines, respectively.

constant $g_{b q}$ incorporated in $M_{q \beta}$ is given by $g_{b q}=g_{b q}^{D}+g_{b q}^{F}$, where $g_{b q}^{D}$ and $g_{b q}^{F}$ represent the coupling constants due to a phenomenological LO-phonon deformation potential interaction and the Fröhlich interaction, respectively. Here, $g_{b q}^{D}$ is real and is considered as approximately independent of $\boldsymbol{q}$, while $g_{b \boldsymbol{q}}^{F}$ is pure imaginary and $\left|g_{b \boldsymbol{q}}^{F}\right| \propto|\boldsymbol{q}|^{-1}$ [108]. In a nonpolar crystal such as $\mathrm{Si}, g_{b q}^{F}$ is ineffective, namely, $g_{b q}=g_{b q}^{D}$, whereas in a polar or partially ionic crystal such as GaAs, $g_{b q}^{F}$ is much dominant to $g_{b q}^{D}$, namely, $g_{b q} \approx g_{b q}^{F}$; the contribution of $g_{b \boldsymbol{q}}^{D}$ to $\bar{A}_{\boldsymbol{q}}\left(t_{p} ; \omega\right)$ is about 1000 times smaller than that of $g_{b q}^{F}$ in our calculations. Due to the approximation 

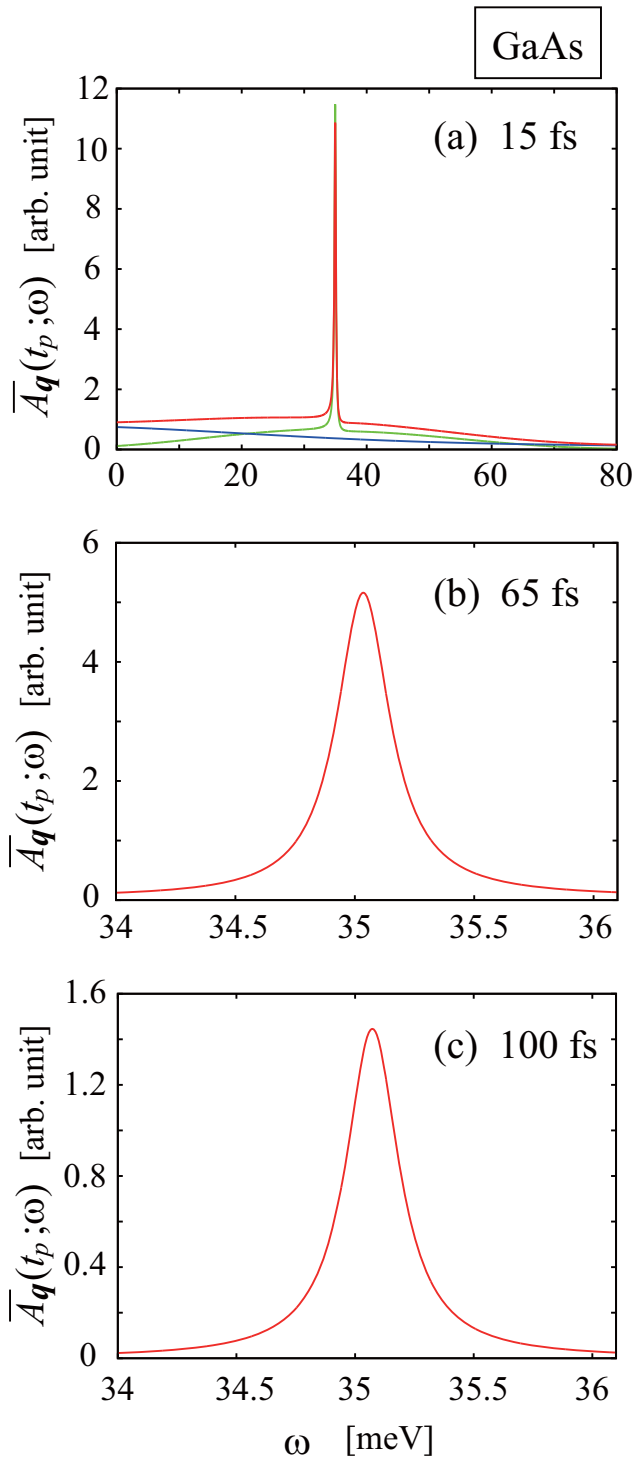

FIG. 4. The same as Fig. 3 but for GaAs.

(ii) described in Sec. II A 4, $U_{\boldsymbol{q} \alpha}^{L^{\dagger}}(\boldsymbol{k} b b)$ is considered real [102] and, thus, the phase of $M_{\boldsymbol{q} \beta}$ is almost determined by that of $g_{b \boldsymbol{q}}$. That is, $M_{\boldsymbol{q} \beta}$ is a complex number given by

$$
M_{\boldsymbol{q} \beta}=\left|M_{\boldsymbol{q} \beta}\right| e^{i \phi_{q \beta}}
$$

in general; $\phi_{q \beta}=0, \pi$ for $\mathrm{Si}$, while $\phi_{q \beta}= \pm \pi / 2$ for GaAs.

Here, one examines how such difference of $M_{\boldsymbol{q}}$ reflects the spectral profile of $\bar{A}_{q}\left(t_{p} ; \omega\right)$, based on the PQ picture developed here. As is shown in Fig. 2, the discrete state $\alpha_{2}$ of LO phonon is embedded in the continuum state $\beta$ of quasiboson, and it is possible that both are resonantly coupled to form the FR state of PQ (a single PQ state [104]). Given this situation, the FR dynamics is schematically shown by the diagram in Fig. 5. It is seen that there are two transition paths for the process: one is a direct path mediated by an optical transition matrix $D_{q \beta}^{(c)}$ from quasiboson state $\beta$ to the PQ ground state [104] and the other is a two-step resonant path mediated by $M_{\boldsymbol{q} \beta}^{*}$ from $\beta$ to LO-phonon state $\alpha_{2}$, followed by a deexcited process mediated by an optical transition matrix $D_{q \alpha_{2}}^{(r)}$ from $\alpha_{2}$ to the PQ ground

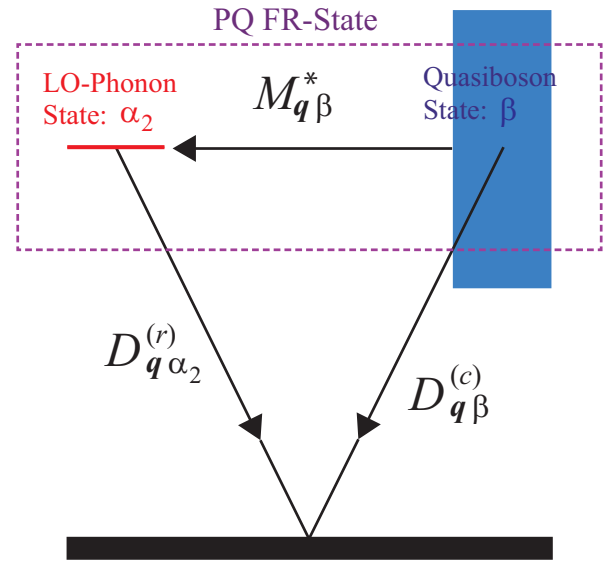

PQ Ground-State

FIG. 5. Schematic diagram of FR dynamics based on the PQ picture, where the LO-phonon state $\alpha_{2}$ is embedded in quasiboson state $\beta$. The PQ FR state composed of $\alpha_{2}$ and $\beta$ is deexcited by induced photoemission process. $D_{q \alpha_{2}}^{(r)}$ and $D_{q \beta}^{(c)}$ represent transition matrices of photoemission from $\alpha_{2}$ and $\beta$ to the PQ ground state, respectively. $M_{q \beta}^{*}$ represents a coupling matrix between $\beta$ and $\alpha_{2}$. For more detail, consult the text.

state. Here, $t_{p}$ dependence of $D_{\boldsymbol{q} \beta}^{(c)}, D_{\boldsymbol{q} \alpha_{2}}^{(r)}$, and $M_{\boldsymbol{q} \beta}^{*}$ is omitted for the sake of simplicity. Owing to Shore's model [109], the whole transition matrix is given by

$$
D_{\boldsymbol{q} \beta}\left(t_{p} ; \omega\right)=D_{\boldsymbol{q} \beta}^{(c)}+\frac{D_{\boldsymbol{q} \alpha_{2}}^{(r)} M_{\boldsymbol{q} \beta}^{*}}{\omega-\omega_{\boldsymbol{q}}^{(\mathrm{LO})}+i \Gamma_{\boldsymbol{q} \alpha_{2}} / 2},
$$

where the natural spectral width is represented by $\Gamma_{\boldsymbol{q} \alpha_{2}}=$ $2 \pi \rho_{\boldsymbol{q} \alpha_{2}}\left|M_{\boldsymbol{q} \alpha_{2}}\right|^{2} ; \rho_{\boldsymbol{q} \alpha_{2}}$ and $M_{\boldsymbol{q} \alpha_{2}}$ are the density of state of quasiboson and the coupling matrix at $\mathcal{E}_{\boldsymbol{q} \beta}=\omega_{\boldsymbol{q}}^{(\mathrm{LO})}$, respectively. The induced photoemission spectra given by $\bar{A}_{q}\left(t_{p} ; \omega\right)=$ $\left|D_{q \beta}\left(t_{p} ; \omega\right)\right|^{2}$ show Shore's spectral profile in the proximity of $\omega \approx \omega_{q}^{(\mathrm{LO})}$, which is equivalent to well-known Fano's formula [109]. That is,

$$
\bar{A}_{\boldsymbol{q}}\left(t_{p} ; \omega\right) \approx \mathcal{C}_{\boldsymbol{q} \beta}+\frac{\mathcal{A}_{\boldsymbol{q} \alpha_{2}}\left(\omega-\omega_{\boldsymbol{q}}^{(\mathrm{LO})}\right)+\mathcal{B}_{\boldsymbol{q} \alpha_{2}} \Gamma_{\boldsymbol{q} \alpha_{2}} / 2}{\left(\omega-\omega_{\boldsymbol{q}}^{(\mathrm{LO})}\right)^{2}+\left(\Gamma_{\boldsymbol{q} \alpha_{2}} / 2\right)^{2}},
$$

where Shore's spectral parameters of $\mathcal{A}_{\boldsymbol{q} \alpha_{2}}, \mathcal{B}_{\boldsymbol{q} \alpha_{2}}$, and $\mathcal{C}_{\boldsymbol{q} \beta}$ are provided by

$$
\begin{gathered}
\mathcal{A}_{\boldsymbol{q} \alpha_{2}}=2\left|D_{\boldsymbol{q} \beta}^{(c)}\right|\left|D_{\boldsymbol{q} \alpha_{2}}^{(r)}\right|\left|M_{\boldsymbol{q} \beta}\right| \cos \tilde{\phi}_{\boldsymbol{q} \beta}, \\
\mathcal{B}_{\boldsymbol{q} \alpha_{2}}=-2\left|D_{\boldsymbol{q} \beta}^{(c)}\right|\left|D_{\boldsymbol{q} \alpha_{2}}^{(r)}\right|\left|M_{\boldsymbol{q} \beta}\right| \sin \tilde{\phi}_{\boldsymbol{q} \beta}+\frac{\left|D_{\boldsymbol{q} \alpha_{2}}^{(r)}\right|^{2}\left|M_{\boldsymbol{q} \beta}\right|^{2}}{\Gamma_{\boldsymbol{q} \alpha_{2}} / 2},
\end{gathered}
$$

and

$$
\mathcal{C}_{\boldsymbol{q} \beta}=\left|D_{\boldsymbol{q} \beta}^{(c)}\right|^{2},
$$

respectively. Here, the phase $\tilde{\phi}_{\boldsymbol{q} \beta}$ is given by $\tilde{\phi}_{\boldsymbol{q} \beta}=$ $\phi_{\boldsymbol{q} \beta}+\Delta \phi_{\boldsymbol{q} \beta}$ with $\Delta \phi_{\boldsymbol{q} \beta}=\arg D_{\boldsymbol{q} \alpha_{2}}^{(c)}-\arg D_{\boldsymbol{q} \beta}^{(r)}$. The associated Fano's $q$ parameter is determined in terms of Shore's 
parameters as

$$
q_{\boldsymbol{q} \alpha_{2}}\left(t_{p}\right)=r_{\boldsymbol{q} \alpha_{2}}\left(t_{p}\right)+\sigma_{\boldsymbol{q} \alpha_{2}}\left(t_{p}\right) \sqrt{\left[r_{\boldsymbol{q} \alpha_{2}}\left(t_{p}\right)\right]^{2}+1}
$$

with $r_{\boldsymbol{q} \alpha_{2}}\left(t_{p}\right)=\mathcal{B}_{\boldsymbol{q} \alpha_{2}} / \mathcal{A}_{\boldsymbol{q} \alpha_{2}}$ and $\sigma_{\boldsymbol{q} \alpha_{2}}\left(t_{p}\right)=\mathcal{A}_{\boldsymbol{q} \alpha_{2}} /\left|\mathcal{A}_{\boldsymbol{q} \alpha_{2}}\right|$.

An asymmetric spectral profile is exclusively determined by $\mathcal{A}_{q \alpha_{2}}$, and this is superimposed with continuum background governed by $\mathcal{C}_{\boldsymbol{q} \beta}$. It is seen that $\mathcal{A}_{\boldsymbol{q}_{\alpha_{2}}}\left(t_{p}\right)$ vanishes for $\tilde{\phi}_{q \beta}= \pm \pi / 2$, and the observed spectra $\bar{A}_{q}\left(t_{p} ; \omega\right)$ become of symmetric shape with $\left|q_{\boldsymbol{q} \alpha_{2}}\left(t_{p}\right)\right|$ infinite. The spectral profile of GaAs shown in Fig. 4(b) corresponds to this case, where $\Delta \phi_{q \beta}$ is considered negligibly small because $\phi_{q \beta}= \pm \pi / 2$. For $\tilde{\phi}_{\boldsymbol{q} \beta} \neq \pm \pi / 2$, both $\mathcal{A}_{\boldsymbol{q} \alpha_{2}}\left(t_{p}\right)$ and $\mathcal{B}_{\boldsymbol{q} \alpha_{2}}\left(t_{p}\right)$ are finite, and $\bar{A}_{\boldsymbol{q}}\left(t_{p} ; \omega\right)$ becomes of asymmetric shape with $\left|q_{\boldsymbol{q} \alpha_{2}}\left(t_{p}\right)\right|$ finite. The spectral profile of Si shown in Fig. 3(b) corresponds to this case, where $\tilde{\phi}_{q \beta} \approx 0, \pi ; \Delta \phi_{q \beta} \approx 0$ is assumed according to GaAs. For Figs. 3(c) and 4(c), since $D_{q \bar{\alpha}}^{(c)}$ and $\left|M_{q \beta}\right|$ become negligibly small, $\bar{A}_{q}\left(t_{p} ; \omega\right)$ is governed by the second term of Eq. (79), and this becomes symmetric with $\Gamma_{q \alpha_{2}} \approx 0$. To conclude, the effective coupling $M_{\boldsymbol{q} \beta}$ around $\mathcal{E}_{\boldsymbol{q} \beta} \approx \omega_{\boldsymbol{q}}^{(\mathrm{LO})}$ plays the crucial role of the manifestation of transient FR, and the asymmetry of profile is mostly determined by $\phi_{\boldsymbol{q} \beta}$ as long as $\left|M_{\boldsymbol{q} \beta}\right|$ is still large.

Next, the optical transition matrix $D_{\boldsymbol{q} \alpha_{2}}^{(r)}$ for the LO phonon is examined. A $\Gamma_{4}$ optical phonon of GaAs, classified to the space group $T_{d}^{2}(F \overline{4} 3 m)$, can be directly excited by an infrared photon via an electric dipole transition, implying that $D_{q \alpha_{2}}^{(r)} \neq 0$. On the other hand, a $\Gamma_{25^{\prime}}$ optical phonon of $\mathrm{Si}$, classified to the space group $O_{h}^{7}(F d 3 m)$, is not infrared active because of the presence of inversion symmetry. However, it is not necessarily true that $D_{\boldsymbol{q} \alpha_{2}}^{(r)}$ vanishes in the present transient and optically nonlinear process. The physical system to be concerned is a crystal with application of a pump laser in a certain direction rather than the free crystal of $\mathrm{Si}$; hereafter, the former crystal is termed as a dressed crystal. For instance, on the occasion that an electric field of the laser is applied in the directions of [111], the symmetry of the dressed crystal is lowered from the original point group $O_{h}$ into point groups of $C_{3 v}$. Irreducible representations subduced from the most concerned irreducible representation $\Gamma_{25^{\prime}}$ of $O_{h}$ to $C_{3 v}$ are readily obtained as follows [110]: $\Gamma_{25^{\prime}} \downarrow \mathrm{C}_{3 \mathrm{v}}=\Lambda_{1}+\Lambda_{3}$. The irreducible representations of $\Lambda_{1}$ and $\Lambda_{3}$ are consistent with the symmetry of ionic momentum operators, that is, $\Lambda_{1}:\{z\}$ and $\Lambda_{3}:\{x \pm i y\}$, where $\Lambda_{1}$ and $\Lambda_{3}$ are single-valued and double-valued representations, respectively. The similar results are obtained for the application of the laser in other directions. Therefore, the dressed crystal can be infrared active and optically deexcited through an emission process induced by an infrared laser, differing from the free crystal, that is, $D_{q \alpha_{2}}^{(r)} \neq 0$ even in $\mathrm{Si}$.

The subduced representations $\Lambda_{1}$ and $\Lambda_{3}$ are in harmony with the compatibility relations with respect to $\Gamma_{25^{\prime}}$ point [110]. To be specific, in the phonon energy-dispersion diagram of Si [108], the $\boldsymbol{k}$ group $\mathcal{G}_{\Lambda}$ relevant to $\Lambda$ point $\left(\boldsymbol{k}_{\Lambda}\right)$ along the (111) axis of Bloch momentum, namely, the direction of the $L$ point, is a subgroup of the $\boldsymbol{k}$ group $\mathcal{G}_{\Gamma}$ relevant to the $\Gamma$ point $\left(\boldsymbol{k}_{\Gamma}=\mathbf{0}\right)$ at the zone center. Here, the symmetry lowering of the dressed crystal in Si from $\boldsymbol{k}_{\Gamma}$ to $\boldsymbol{k}_{\Lambda}$ along the (111) axis makes a threefold-degenerate level $\Gamma_{25}$ lifted into nondegenerate level $\Lambda_{1}$ and a twofold-degenerate level $\Lambda_{3}$. According to this discussion, the degree of magnitude of the symmetry lowering from $\Gamma_{25^{\prime}}$ to $\Lambda_{1}$ is comparable to the momentum change of $|\boldsymbol{q}|$ with $\boldsymbol{q} \equiv \boldsymbol{k}_{\Lambda}-\boldsymbol{k}_{\Gamma}$. In reality, this change of momentum results from spatial inhomogeneity entailed by the formation of polarized charge induced by the pump laser $[12,13,55,56]$. That is, the generation of such polarization leads to the breaking of spatial inversion symmetry. For this reason, the intensity of $\bar{A}_{\boldsymbol{q}}\left(t_{p} ; \omega\right)$ due to the contribution from $\tilde{\chi}_{\boldsymbol{q}}^{\prime}\left(t_{p} ; \omega\right)$ in $\mathrm{Si}$ is reduced by the order of $|\boldsymbol{q}|^{2}$ compared with that in GaAs (see Figs. 3 and 4 ).

Therefore, $\tilde{\chi}_{q}^{\prime}\left(t_{p} ; \omega\right)$ is governed by an electric-dipole transition in the dressed crystal with absorption of, for instance, a $\Lambda_{1}$ optical phonon. It is also stated that this is also governed by an electric quadrupole transition in the original crystal with absorption of a $\Gamma_{25^{\prime}}$ optical phonon, as long as $\boldsymbol{q} \approx \mathbf{0}$; actually, the irreducible representation is consistent with the symmetry $\{x y, y z, z x\}[108,110]$. Significant roles of the electric quadrupole transition are also addressed in optical second-harmonic generation from $\mathrm{Si}$ [111] and lattice instability of $\mathrm{Si}$ and $\mathrm{GaAs}$ induced by a strong fs laser pulse due to LO-phonon distortions [112]. The results thus obtained from the group-theoretical point of view remain unaltered in the case of a cubic crystal modeled in the actual calculations of spectra.

Lastly, the allocation of time constants shown in Fig. 1 is discussed, in particular, with reference to $\bar{A}_{q}\left(t_{p} ; \omega\right)$ of Si. This is a significant issue for deepening the understanding of the manifestation of the transient FR. As shown in Fig. 3(b), in the region of $T_{q 12} \lesssim t_{p}<T_{12}$, the asymmetric spectral profile of FR bursts into view from the structureless continuum due to $\tilde{\chi}_{\boldsymbol{q}}\left(t_{p} ; \omega\right)$. Actually, in the early-time region of $t_{p}<T_{12}$, the excited-carrier density is still populated enough around the energy region of $\omega_{\boldsymbol{q}}^{(\mathrm{LO})}$, namely, $\mathcal{E}_{\boldsymbol{q} \beta} \approx \omega_{\boldsymbol{q}}^{(\mathrm{LO})}$, to couple strongly with LO phonon through $M_{\boldsymbol{q} \alpha_{2}}$ to form the FR spectra. Contrarily, the effect of $\tilde{\chi}_{\boldsymbol{q}}\left(t_{p} ; \omega\right)$ is much reduced in the region of $T_{q 12} \lesssim t_{p}$. Allocating a different sequence of time constants, for instance, such that $T_{q 12}$ is close to $T_{12}$, namely, $T_{q 12} \sim T_{12}$, in the region of $t_{p}<T_{12}$, the FR profile is no longer discernible since this is covered with still dominant contributions from $\tilde{\chi}_{\boldsymbol{q}}\left(t_{p} ; \omega\right)$, and in the region of $t_{p} \approx T_{12}$, the effect of $M_{\boldsymbol{q} \beta}$ is too small to cause FR. Thus, the allocation of time constants shown in Fig. 1 is regarded as a necessary condition for realizing the FR of Si in $\bar{A}_{\boldsymbol{q}}\left(t_{p} ; \omega\right)$; otherwise, this never appears.

\section{Power spectra of LO-phonon displacement function}

Although $\bar{A}_{q}\left(t_{p}, \omega\right)$ are directly concerned in the change of induced electron density at time $t_{p}$, it is considered difficult to extract the transient FR from the spectra of $\mathrm{Si}$ in actual measurements since the magnitude of $\tilde{\chi}_{q}^{\prime}\left(t_{p} ; \omega\right)$ is much reduced by the order of $|\boldsymbol{q}|^{2}$ compared with that of $\tilde{\chi}_{\boldsymbol{q}}\left(t_{p} ; \omega\right)$, as stated above. To remedy such inconvenience, one examines an LO-phonon displacement function that is more suitable for experiments, and this is compared with $\tilde{\chi}_{q}^{\prime}\left(t_{p} ; \omega\right)$ in a direct manner. The Fourier transforms of both the phonon displacement function $\tilde{Q}_{q}(\omega)$ and the related power spectra $S_{q}(\omega)$ as functions of frequency $\omega$ are taken into account [see Eqs. (72) and (73)]. 


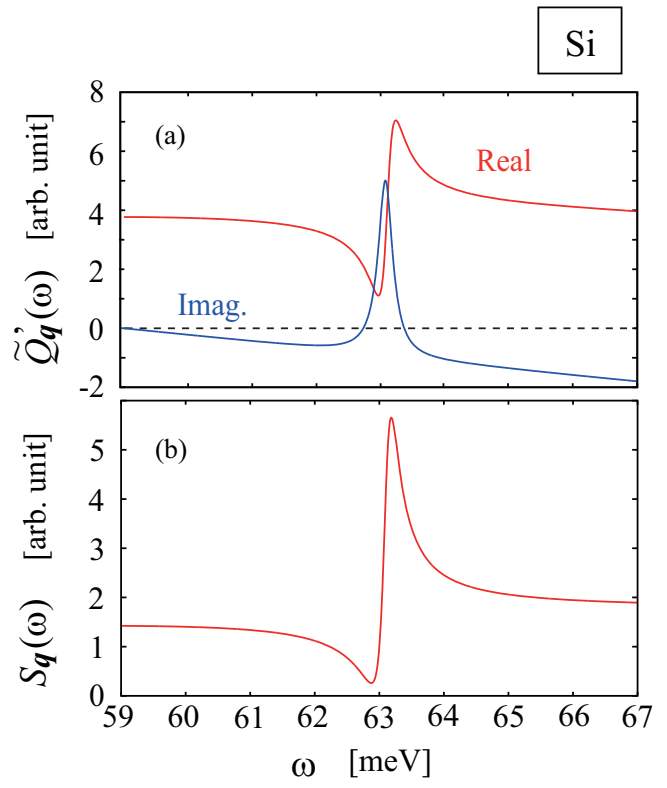

FIG. 6. (a) The Fourier transform of phonon displacement function $\tilde{Q}_{q}^{\prime}(\omega)$ and (b) the power spectra $S_{q}(\omega)$ of Si as a function of frequency $\omega$ (in the unit of $\mathrm{meV}$ ).

For $\omega>0$, the second term $\left\langle c_{-q}^{\dagger}(t)\right\rangle$ in the angled brackets in the right-hand side of Eq. (69) dominantly contributes to $\tilde{Q}_{q}(\omega)$, and the expression of it is given by the second equation of Eq. (41). On the other hand, the second term $\left[\bar{D}_{-q}^{\prime R}\left(t, t_{p}\right)\right]^{*}$ in the right-hand side of Eq. (66) dominantly contributes to $\tilde{\chi}_{q}^{\prime}\left(t_{p} ; \omega\right)$, and the expression of it is given by Eq. (68). It is seen that the expression of $\left\langle c_{-q}^{\dagger}(t)\right\rangle$ has similar $t$ dependence to that of $\left[\bar{D}_{-q}^{\prime R}\left(t, t_{p}\right)\right]^{*}$. Differing from $\tilde{Q}_{q}(\omega)$ that is obtained by the integration over $t$ as shown in Eq. (72), $i \tilde{\chi}_{q}^{\prime}\left(t_{p} ; \omega\right)$ is provided by the Fourier transform of $i \chi_{\boldsymbol{q}}^{\prime}\left(t, t_{p}\right)$ over $\tau=t-t_{p}$. Therefore, at $t_{p}=t_{D}, \tilde{Q}_{q}(\omega)$ becomes almost the same expression as $i \tilde{\chi}_{q}^{\prime}\left(t_{D} ; \omega\right)$ aside from an overall phase factor $e^{i \xi_{q}}$ and a proportionality constant $\lambda_{\boldsymbol{q}}$, where $t_{D}$ is set approximately equal to zero, and both $\xi_{\boldsymbol{q}}$ and $\lambda_{q}$ are real numbers. That is, $\tilde{Q}_{q}^{\prime}(\omega)=i \tilde{\chi}_{q}^{\prime}\left(t_{D} ; \omega\right)$, where $\tilde{Q}_{q}^{\prime}(\omega)=\lambda_{q} e^{i \xi_{q}} \tilde{Q}_{q}(\omega)$. Thus, the following relation as

$$
\operatorname{Re} \tilde{Q}_{q}^{\prime}(\omega)=-\operatorname{Im} \tilde{\chi}_{q}^{\prime}\left(t_{D} ; \omega\right)
$$

is obtained. The power spectra are independent of $\xi_{q}$, given by $S_{q}(\omega) \propto\left|\tilde{Q}_{q}^{\prime}(\omega)\right|^{2}=\left|\lambda_{q} \tilde{Q}_{q}(\omega)\right|^{2}$.

Figure 6(a) shows $\operatorname{Re} \tilde{Q}_{q}^{\prime}(\omega)$ and $\operatorname{Im} \tilde{Q}_{q}^{\prime}(\omega)$ of Si. It is seen that the resulting profile of $\operatorname{Re} \tilde{Q}_{q}^{\prime}(\omega)$ is in good agreement with that of $-\operatorname{Im} \tilde{\chi}_{q}^{\prime}\left(t_{p} ; \omega\right)$ shown in Fig. $3(\mathrm{~b})$ at $t_{p}=65 \mathrm{fs}$, and Eq. (82) still holds approximately:

$$
\operatorname{Re} \tilde{Q}_{q}^{\prime}(\omega) \approx-\operatorname{Im} \tilde{\chi}_{q}^{\prime}\left(t_{p} ; \omega\right) .
$$

Here, the asymmetric profile of $\bar{A}_{q}\left(t_{p}, \omega\right)$ is governed by $-\operatorname{Im} \tilde{\chi}_{q}^{\prime}\left(t_{p} ; \omega\right)$. Actually, this relation is correct as long as the condition of $t_{p}<T_{12}$ is fulfilled. Otherwise, this is not the case, as is seen in Fig. 3(c), at $t_{p}=100$ fs:

$$
\operatorname{Re} \tilde{Q}_{q}^{\prime}(\omega) \neq-\operatorname{Im} \tilde{\chi}_{q}^{\prime}\left(t_{p} ; \omega\right) .
$$

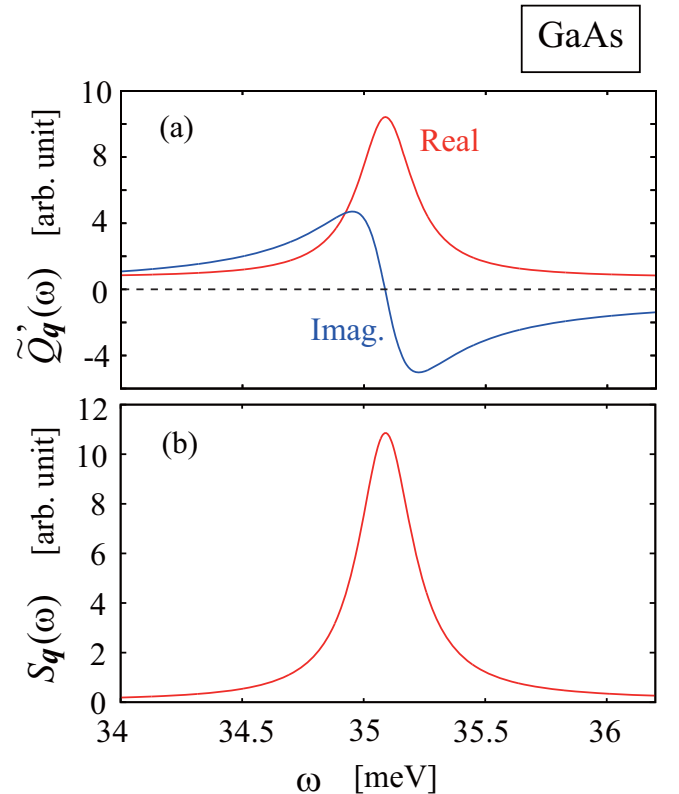

FIG. 7. The same as Fig. 6 but for GaAs.

Therefore, $\operatorname{Re} \tilde{Q}_{q}^{\prime}(\omega)$ reflects properly the transient FR dynamics accompanied by the CP generation occurring in the early-time region without being masked by the contribution from $-\operatorname{Im} \tilde{\chi}_{\boldsymbol{q}}\left(t_{p} ; \omega\right)$ that is dominant in the region of $t_{p} \lesssim T_{q 12}$.

Figure 6(b) shows $S_{q}(\omega)$ as a function of $\omega$, and the overall feature of the spectral profile of $S_{q}(\omega)$ somewhat succeeds to that of $-\operatorname{Im} \tilde{\chi}_{\boldsymbol{q}}^{\prime}\left(t_{p} ; \omega\right)$ at $t_{p}=65$ fs. Aside from the close resemblance between $\operatorname{Re} \tilde{Q}_{q}^{\prime}(\omega)$ and $-\operatorname{Im} \tilde{\chi}_{q}^{\prime}\left(t_{p} ; \omega\right)$ as shown in Eq. (83), it is remarked that the detail of the transient FR dynamics is not always reflected accurately on $S_{q}(\omega)$ if there is a relatively large contribution from $\operatorname{Im} \tilde{Q}_{q}^{\prime}(\omega)$ to $S_{q}(\omega)$. In other words, the parameter $q_{\boldsymbol{q} \alpha_{2}}\left(t_{p}\right)$ of Eq. (81) is not always identical with an asymmetry parameter extracted from $S_{q}(\omega)$. It is $q_{q \alpha_{2}}\left(t_{p}\right)$ that is characteristic of the spectral pattern of $-\operatorname{Im} \tilde{\chi}_{q}^{\prime}\left(t_{p} ; \omega\right)$ at each $t_{p}$ with the firm physical meaning described by Fig. 5, rather than the asymmetry parameter thus extracted.

$\operatorname{Re} \tilde{Q}_{q}^{\prime}(\omega)$ and $\operatorname{Im} \tilde{Q}_{q}^{\prime}(\omega)$ for GaAs are shown in Fig. 7(a) as a function of $\omega$. The spectral profile of $\operatorname{Re} \tilde{Q}_{q}^{\prime}(\omega)$ is symmetric, and, similarly to the case of $\mathrm{Si}$, this looks akin to the profile of $-\operatorname{Im} \tilde{\chi}_{q}^{\prime}\left(t_{p} ; \omega\right)$ shown not only in Fig. 4(b) at $t_{p}=65$ fs but also in Fig. 4(c) at $t_{p}=100$ fs. Figure 7(b) shows $S_{q}(\omega)$ as a function of $\omega$. Since $\operatorname{Re} \tilde{Q}_{q}^{\prime}(\omega)$ and $\operatorname{Im} \tilde{Q}_{q}^{\prime}(\omega)$ are even and odd functions with respect to $\omega=\omega_{q}^{(\mathrm{LO})}$, respectively, $S_{q}(\omega)$ remains of the symmetric shape.

\section{Comparison with other studies}

This work is compared with the three studies by Hase et al. [13], Lee et al. [57], and Riffe [61]. One begins with a comparison with the pioneering work by Hase et al., in which transient electrooptic reflectivity signals due to the $\mathrm{CP}$ generation were measured in a lightly $n$-doped Si crystal [13]. In the associated continuous-wavelet-transformed spectra, an asymmetric profile was found around 50 fs immediately after the onset of the irradiation of pump pulse. This conspicuous phenomenon was suggestive of the transient manifestation 
of quantum interference between excited electrons and an LO phonon leading to FR. Further, given the situation that this coupling was such a strong interaction that the electron and phonon were considered as a composite particle, the authors supposed the birth of PQ. The present theoretical model is based on this supposition, although this was naive. As illustrated in Sec. III, the PQ picture thus developed can successfully explain the manifestation of the FR.

The transient reflectivity signals accompanying CP generation were observed in the experimentally setup $\Gamma_{25^{\prime}}$ geometry. This geometry would correspond to the $\Lambda_{1}$ irreducible representation in the symmetry $\Gamma_{25^{\prime}} \downarrow C_{3 v}$ given in Sec. III B. This vibrational state of dressed crystal can be optically deexcited through an emission process induced by an infrared laser polarized in the [001] direction. In the actual measurements, CP signals are caused by a lot of carrier transitions along the $\Lambda$ and $\Delta$ directions in the Brillouin zone as well as near the $\Gamma$ point. Since the energy dispersions of conduction bands along the $\Lambda$ ( $L$ valley) direction and the $\Delta$ ( $X$ valley) direction are almost parallel to the energy dispersion of the heavy-hole valence band, relative energy of an electron-hole pair, given by $w_{c v k q}$ of Eq. (14), is almost dispersionless, namely, independent of $\boldsymbol{k}$. Thus, it is speculated that just the electron excitation near the $\Gamma$ point forms broad band spectra due to electron-hole continuum states with $\boldsymbol{k}$ dispersion, giving rise to transient FR; this differs a lot from the transitions along the $\Lambda$ and $\Delta$ directions.

The calculated results based on the transient PQ picture seem in harmony to a certain extent with the experimental results of Hase et al. [113]. Nevertheless, as things now stand, this fact does not necessarily imply that the PQ introduced here is a real entity. This issue is beyond the scope of this study, though quite challenging.

Lee et al. presented the first theoretical approach to the FR problem associated with the CP generation [57]. They calculated a phonon displacement function similar to $Q_{q}(t)$ by solving time-dependent Schrödinger equations for the system of GaAs. An asymmetric line shape was obtained in the continuous-wavelet-transformed spectra of the associated time signals. Further, they interpreted the underlying dynamics causing the FR as quantum interference between two diagrams with different time ordering in the one-phonon Raman process. These results differ from those obtained here in that in the latter, the transient FR never takes place in $S_{q}(\omega)$ relevant to $Q_{q}(t)$ in GaAs and that the origin of FR observed in $\mathrm{Si}$ is not the interference between the Raman diagrams; thus far, FR spectra have not been observed in GaAs in the existing experiments [11,114].

Riffe proposed a classical Fano oscillator model based on the Fano-Anderson Hamiltonian, in which a set of coupled oscillators are driven by as many fictitious external forces introduced a posteriori [61]. According to this study, this Hamiltonian reads as

$$
\begin{aligned}
\hat{H}_{\boldsymbol{q}}^{(\mathrm{FA})}= & \omega_{\boldsymbol{q}}^{(\mathrm{LO})} c_{\boldsymbol{q}}^{\dagger} c_{\boldsymbol{q}}+\sum_{\beta} \mathcal{E}_{\boldsymbol{q} \beta} B_{\boldsymbol{q} \beta}^{\dagger} B_{\boldsymbol{q} \beta} \\
& +\sum_{\beta}\left(M_{\boldsymbol{q} \beta} c_{\boldsymbol{q}} B_{\boldsymbol{q} \beta}^{\dagger}+M_{\boldsymbol{q} \beta}^{*} c_{\boldsymbol{q}}^{\dagger} B_{\boldsymbol{q} \beta}\right),
\end{aligned}
$$

where the quasiboson is considered as a real boson with real eigenenergy $\mathcal{E}_{\boldsymbol{q} \beta}$. Thus, defining displacement functions relevant to quasiboson and LO-phonon operators as $X_{q}^{(+)}(t)=$ $\left\langle c_{\boldsymbol{q}}(t)+c_{-\boldsymbol{q}}^{\dagger}(t)\right\rangle / 2$ and $x_{\boldsymbol{q} \beta}^{(+)}(t)=\left\langle B_{\boldsymbol{q} \beta}(t)+B_{-\boldsymbol{q} \beta}^{\dagger}(t)\right\rangle / 2$, respectively, the associated equations of motion are readily obtained from Eq. (85). Let external forces associated with $X_{\boldsymbol{q}}^{(+)}(t)$ and $x_{\boldsymbol{q} \beta}^{(+)}(t)$ be denoted as $\mathcal{F}_{\boldsymbol{q}}(t)$ and $f_{\boldsymbol{q} \beta}(t)$, respectively. These forces just correspond to the fictitious forces introduced by Riffe, which resulted in FR. According to the present PQ model, the main origin of these forces can be identified as the time derivative of quasiboson adiabatic energy $d \mathcal{E}_{\boldsymbol{q} \beta}(t) / d t$, the time derivative of the effective coupling $d M_{\boldsymbol{q} \beta}(t) / d t$, and the nonadiabatic coupling $W_{\boldsymbol{q} \beta \beta^{\prime}}(t)$ of Eq. (27). In Riffe's work, $\mathcal{E}_{\boldsymbol{q} \beta}$ and $M_{\boldsymbol{q} \beta}$ were assumed independent of time $t$ in addition to $X_{\boldsymbol{q}}^{(+)}(t)$ and $x_{q \beta}^{(+)}(t)$ being regarded as expectation values with respect to a coherent state. This assumption is brought to the result that all of $d \mathcal{E}_{\boldsymbol{q} \beta}(t) / d t$, $d M_{\boldsymbol{q} \beta}(t) / d t$, and $W_{\boldsymbol{q} \beta \beta^{\prime}}(t)$ vanish simultaneously. Eventually, $\mathcal{F}_{\boldsymbol{q}}(t)$ and $f_{\boldsymbol{q} \beta}(t)$ become residual forces just attributed to frictional forces due to an effective damping factor $\gamma_{\boldsymbol{q} \beta \beta^{\prime}}^{(B)}(t)$ of Eq. (28), that is, $\mathcal{F}_{\boldsymbol{q}}(t) \approx-\sum_{\beta, \beta^{\prime}} x_{\boldsymbol{q} \beta}^{(+)}(t) \operatorname{Im}\left[\gamma_{\boldsymbol{q} \beta \beta^{\prime}}^{(B)}(t) M_{\boldsymbol{q} \beta^{\prime}}\right] / 2$ and $f_{\boldsymbol{q} \beta}(t) \approx-\mathcal{E}_{\boldsymbol{q} \beta} \sum_{\beta^{\prime}} x_{\boldsymbol{q} \beta^{\prime}}^{(+)}(t) \operatorname{Im} \gamma_{\boldsymbol{q} \beta^{\prime} \beta}^{(B)}(t) / 2$, respectively. In particular, in $\mathrm{Si}$, given the fact that $M_{\boldsymbol{q} \beta^{\prime}}$ and $\gamma_{\boldsymbol{q} \beta \beta^{\prime}}^{(B)}(t)$ are real, both forces end up with $\mathcal{F}_{\boldsymbol{q}}(t) \approx 0$ and $f_{\boldsymbol{q} \beta}(t) \approx 0$. Therefore, Riffe's results seem incompatible with the results based on the adiabatic picture of the PQ model.

\section{CONCLUSIONS}

The fully quantum-mechanical picture for the $\mathrm{CP}$ generation dynamics is constructed based on the PQ model, and is applied to the longitudinal susceptibility $\tilde{\chi}_{q}^{(t)}\left(t_{p} ; \omega\right)$ in order to shed light on the detail of transient and optically nonlinear FR. The associated photoemission spectra $\bar{A}_{q}\left(t_{p} ; \omega\right)$ are calculated, which reveal the temporal change of spectra due to the strongly excited electron-hole continuum coupled with LO phonon. The present model succeeds in demonstrating the appearance of asymmetric spectral profile in $\mathrm{Si}$ in a flash in the temporal region of $T_{q 12} \lesssim t_{p}<T_{12}$, whereas the profile observed in GaAs is always symmetric. Such difference between $\mathrm{Si}$ and GaAs originates from the effective coupling $M_{\boldsymbol{q} \beta}\left(t_{p}\right)$; the asymmetry is mostly determined by $\arg M_{\boldsymbol{q} \beta}\left(t_{p}\right)$ in the early-time region of $t_{p}<T_{12}$ when $\left|M_{q \beta}\left(t_{p}\right)\right|$ is still strong. Details of the dependence of $M_{q \alpha}\left(t_{p}\right)$ on the spectral profile of $\bar{A}_{\boldsymbol{q}}\left(t_{p} ; \omega\right)$ are analyzed by means of Shore's model and the group theory.

The PQ model is also applied to the LO-phonon displacement function $\tilde{Q}_{q}(\omega)$ and the power spectra $S_{q}(\omega)$. There is approximate equivalence in FR spectral shape between the scaled LO-phonon displacement function $\tilde{Q}_{q}^{\prime}(\omega)$ and the longitudinal susceptibility due to LO-phonon-induced interaction $\tilde{\chi}_{q}^{\prime}\left(t_{p} ; \omega\right)$ just in the early-time region: $\operatorname{Re} \tilde{Q}_{q}^{\prime}(\omega) \approx$ $-\operatorname{Im} \tilde{\chi}_{q}^{\prime}\left(t_{p} ; \omega\right)$. The spectral profile of $S_{q}(\omega)$ succeeds somewhat to that of $-\operatorname{Im} \tilde{\chi}_{q}^{\prime}\left(t_{p} ; \omega\right)$. The present model is nicely in accord with the experimental results obtained by Hase et al. [13], although not consistent with the existing theoretical results $[57,61]$.

To conclude, it is found that in order to realize the transient FR in the $\mathrm{CP}$ dynamics, the following conditions 
are to be fulfilled simultaneously. First, the coupling of an LO phonon with an electron-hole continuum is conducted by the LO-phonon deformation potential interaction rather than by the Fröhlich interaction, as in the case of Si. Second, photoexcited carriers are populated enough around the energy region $\mathcal{E}_{\boldsymbol{q} \beta} \approx \omega_{\boldsymbol{q}}^{(\mathrm{LO})}$ to couple with an LO phonon strongly and resonantly in the early-time region. As a result, the adiabatic-energy configuration shown in Fig. 2 is formed. Third, for extracting the FR profile from $\bar{A}_{q}\left(t_{p} ; \omega\right)$ in $\mathrm{Si}$, it is required that various time constants line up in the order shown in Fig. 1 with $T_{q 12} \ll T_{12}$. Finally, it is pointed out that the present PQ model allows one to extract an $a b$ initio expression of an initial phase from $Q_{q}(t)$ without consulting both the ISRS model and the DECP model.

\section{ACKNOWLEDGMENT}

This work was supported by JSPS KAKENHI Grants No. JP23540360 and No. JP15K05121.
[1] Ultrafast Phenomena XIX: Proceedings of the 19th International Conference, Springer Proceedings in Physics Vol. 162, edited by K. Yamanouchi, S. Cundiff, R. de Vivie-Riedle, M. Kuwata-Gonokami, and L. DiMauro (Springer, Berlin, 2015).

[2] T. Dekorsy, G. C. Cho, and H. Kurz, in Light Scattering in Solids VIII: Fullerens, Semiconductor Surfaces, Coherent Phonons, Topics in Applied Physics Vol. 76, edited by M. Cardona and G. Güntherodt (Springer, Berlin, 2000), Chap. 4.

[3] A. V. Kuznetsov and C. J. Stanton, in Ultrafast Phenomena in Semiconductors, edited by K. T. Tsen (Springer, Berlin, 2001), Chap. 7.

[4] A. V. Kuznetsov and C. J. Stanton, Phys. Rev. Lett. 73, 3243 (1994).

[5] G. C. Cho, W. Kütt, and H. Kurz, Phys. Rev. Lett. 65, 764 (1990).

[6] T. Pfeifer, T. Derkosy, W. Kütt, and H. Kurz, Appl. Phys. A: Solids Surf. 55, 482 (1992).

[7] T. Dekorsy, T. Pfeifer, W. Kütt, and H. Kurz, Phys. Rev. B 47, 3842 (1993).

[8] T. Dekorsy, H. Kurz, X. Q. Zhou, and K. Ploog, Appl. Phys. Lett. 63, 2899 (1993).

[9] G. C. Cho, H. J. Bakker, T. Dekorsy, and H. Kurz, Phys. Rev. B 53, 6904 (1996).

[10] Y. M. Chang, L. Xu, and H. W. K. Tom, Phys. Rev. Lett. 78, 4649 (1997).

[11] O. V. Misochko, J. Exp. Theor. Phys. 92, 246 (2001).

[12] A. J. Sabbah and D. M. Riffe, Phys. Rev. B 66, 165217 (2002).

[13] M. Hase, M. Kitajima, A. M. Constantinescu, and H. Petek, Nature (London) 426, 51 (2003).

[14] D. M. Riffe and A. J. Sabbah, Phys. Rev. B 76, 085207 (2007).

[15] K. Kato, A. Ishizawa, K. Oguri, K. Tateno, T. Tawara, H. Gotoh, M. Kitajima, and H. Nakano, Jpn. J. Appl. Phys. 48, 100205 (2009).

[16] K. A. Nelson, D. D. Dlott, and M. D. Fayer, Chem. Phys. Lett. 64, 88 (1979).

[17] H. J. Bakker, S. Hunsche, and H. Kurz, Phys. Rev. Lett. 69, 2823 (1992).

[18] H. J. Bakker, S. Hunsche, and H. Kurz, Phys. Rev. B 50, 914 (1994).

[19] H. J. Bakker, S. Hunsche, and H. Kurz, Rev. Mod. Phys. 70, 523 (1998).

[20] T. K. Cheng, S. D. Brorson, A. S. Kazeroonian, J. S. Moodera, G. Dresselhaus, M. S. Dresselhaus, and E. P. Ippen, Appl. Phys. Lett. 57, 1004 (1990).

[21] T. K. Cheng, J. Vidal, H. J. Zeiger, G. Dresselhaus, M. S. Dresselhaus, and E. P. Ippen, Appl. Phys. Lett. 59, 1923 (1991).
[22] T. Dekorsy, H. Auer, C. Waschke, H. J. Bakker, H. G. Roskos, H. Kurz, V. Wagner, and P. Grosse, Phys. Rev. Lett. 74, 738 (1995).

[23] S. Hunsche, K. Wienecke, T. Dekorsy, and H. Kurz, Phys. Rev. Lett. 75, 1815 (1995).

[24] M. Hase, K. Mizoguchi, H. Harima, S. Nakashima, M. Tani, K. Sakai, and M. Hangyo, Appl. Phys. Lett. 69, 2474 (1996).

[25] M. Hase, K. Mizoguchi, H. Harima, S. I. Nakashima, and K. Sakai, Phys. Rev. B 58, 5448 (1998).

[26] M. Hase, K. Ishioka, M. Kitajima, K. Ushida, and S. Hishita, Appl. Phys. Lett. 76, 1258 (2000).

[27] O. V. Misochko, K. Sakai, and S. I. Nakashima, Phys. Rev. B 61, 11225 (2000).

[28] M. F. DeCamp, D. A. Reis, P. H. Bucksbaum, and R. Merlin, Phys. Rev. B 64, 092301 (2001).

[29] M. Hase, M. Kitajima, S. I. Nakashima, and K. Mizoguchi, Phys. Rev. Lett. 88, 067401 (2002).

[30] O. V. Misochko, M. Hase, K. Ishioka, and M. Kitajima, Phys. Rev. Lett. 92, 197401 (2004).

[31] É. D. Murray, D. M. Fritz, J. K. Wahlstrand, S. Fahy, and D. A. Reis, Phys. Rev. B 72, 060301(R) (2005).

[32] O. V. Misochko, K. Ishioka, M. Hase, and M. Kitajima, J. Phys.: Condens. Matter 19, 156227 (2007).

[33] O. V. Misochko and M. V. Lebedeva, J. Exp. Theor. Phys. 120, 651 (2015).

[34] M. Hase, K. Ishioka, J. Demsar, K. Ushida, and M. Kitajima, Phys. Rev. B 71, 184301 (2005).

[35] M. Hase, J. Demsar, and M. Kitajima, Phys. Rev. B 74, 212301 (2006).

[36] J. J. Li, J. Chen, D. A. Reis, S. Fahy, and R. Merlin, Phys. Rev. Lett. 110, 047401 (2013).

[37] J. M. Chwalek, C. Uher, J. F. Whitaker, G. Mourou, J Agostinelli, and. M. Lelental, Appl. Phys. Lett. 57, 1696 (1990).

[38] J. M. Chwalek, C. Uher, J. F. Whitaker, G. A. Mourou, and J. Agostinelli, Appl. Phys. Lett. 58, 980 (1991).

[39] W. Albrecht, Th. Kruse, and H. Kurz, Phys. Rev. Lett. 69, 1451 (1992).

[40] O. V. Misochko, Phys. Lett. A 269, 97 (2000).

[41] O. V. Misochko, K. Kisoda, K. Sakai, and S. Nakashima, Phys. Rev. B 61, 4305 (2000).

[42] I. Bozovic, M. Schneider, Y. Xu, R. Sobolewski, Y. H. Ren, G. Lüpke, J. Demsar, A. J. Taylor, and M. Onellion, Phys. Rev. B 69, 132503 (2004).

[43] T. Mishina, K. Nitta, and Y. Masumoto, Phys. Rev. B 62, 2908 (2000). 
[44] K. Ishioka, M. Hase, M. Kitajima, and K. Ushida, Appl. Phys. Lett. 78, 3965 (2001).

[45] K. Watanabe, N. Takagi, and Y. Masumoto, Chem. Phys. Lett. 366, 606 (2002).

[46] K. Watanabe, N. Takagi, and Y. Matsumoto, Phys. Rev. Lett. 92, 057401 (2004).

[47] Y.-X. Yan, E. B. Gamble, and K. Nelson, J. Chem. Phys. 83, 5391 (1985).

[48] Y.-X. Yan and K. Nelson, J. Chem. Phys. 87, 6240 (1987).

[49] W. Kütt, W. Albrecht, and H. Kurz, IEEE J. Quantum Electron. 28, 2434 (1992).

[50] H. J. Zeiger, J. Vidal, T. K. Cheng, E. P. Ippen, G. Dresselhaus, and M. S. Dresselhaus, Phys. Rev. B 45, 768 (1992).

[51] G. A. Garrett, T. F. Albrecht, J. F. Whitaker, and R. Merlin, Phys. Rev. Lett. 77, 3661 (1996).

[52] R. Merlin, Solid State Commun. 102, 207 (1997).

[53] T. E. Stevens, J. Kuhl, and R. Merlin, Phys. Rev. B 65, 144304 (2002).

[54] A. V. Bragas, C. Aku-Leh, S. Costantino, A. Ingale, J. Zhao, and R. Merlin, Phys. Rev. B 69, 205306 (2004).

[55] T. Pfeifer, W. Kütt, H. Kurz, and R. Scholz, Phys. Rev. Lett. 69, 3248 (1992).

[56] R. Scholz, T. Pfeifer, and H. Kurz, Phys. Rev. B 47, 16229 (1993).

[57] J. D. Lee, J. Inoue, and M. Hase, Phys. Rev. Lett. 97, 157405 (2006).

[58] Y. Shinohara, K. Yabana, Y. Kawashita, J. I. Iwata, T. Otobe, and G. F. Bertsch, Phys. Rev. B 82, 155110 (2010).

[59] G. D. Mahan, Many-Particle Physics (Plenum, New York, 1981), Chaps. 4 and 5.

[60] U. Fano, Phys. Rev. 124, 1866 (1961).

[61] D. M. Riffe, Phys. Rev. B 84, 064308 (2011).

[62] K. G. Nakamura, Y. Shikano, and Y. Kayanuma, Phys. Rev. B 92, 144304 (2015)

[63] P. Gaal, W. Kuehn, K. Reimann, M. Woerner, T. Elsaesser, and R. Hey, Nature (London) 450, 1210 (2007).

[64] S. Yoshino, G. Oohata, and K. Mizoguchi, Phys. Rev. Lett. 115, 157402 (2015).

[65] B. B. Varga, Phys. Rev. 137, A1896 (1965).

[66] A. Mooradian and A. L. McWhorter, Phys. Rev. Lett. 19, 849 (1967).

[67] M. V. Klein, in Light Scattering in Solids I: Introductory Concepts, Topics in Applied Physics Vol. 8, edited by M. Cardona (Springer, Berlin, 1983), Chap. 4.

[68] A. V. Kuznetsov and C. J. Stanton, Phys. Rev. B 51, 7555 (1995).

[69] M. Hase, S.-i. Nakashima, K. Mizoguchi, H. Harima, and K. Sakai, Phys. Rev. B 60, 16526 (1999).

[70] K. Ishioka, A. K. Basak, and H. Petek, Phys. Rev. B 84, 235202 (2011).

[71] J. Hu, O. V. Misochko, A. Goto, and K. G. Nakamura, Phys. Rev. B 86, 235145 (2012).

[72] R. Huber, C. Kübler, S. Tübel, A. Leitenstorfer, Q. T. Vu, H. Haug, F. Köhler, and M.-C. Amann, Phys. Rev. Lett. 94, 027401 (2005).

[73] Y.-M. Chang, in Conference on Lasers and ElectroOptics/Quantum Electronics and Laser Science Conference 2010 Technical Digest (Optical Society of America, Washington, DC, 2010), QThF1.

[74] J. P. Russell, Appl. Phys. Lett. 6, 223 (1965).
[75] J. H. Parker, Jr., D. W. Feldman, and M. Ashkin, Phys. Rev. 155, 712 (1967).

[76] T. R. Hart, R. L. Aggarwal, and B. Lax, Phys. Rev. B 1, 638 (1970).

[77] F. Cerdeira, T. A. Fjeldy, and M. Cardona, Solid State Commum. 8, 133 (1970).

[78] F. Cerdeira and M. Cardona, Phys. Rev. B 5, 1440 (1972).

[79] F. Cerdeira, T. A. Fjeldy, and M. Cardona, Solid State Commum. 13, 325 (1973).

[80] P. A. Temple and C. E. Hathaway, Phys. Rev. B 7, 3685 (1973).

[81] F. Cerdeira, T. A. Fjeldy, and M. Cardona, Phys. Rev. B 8, 4734 (1973).

[82] F. Cerdeira, T. A. Fjeldy, and M. Cardona, Phys. Rev. B 9 , 4344 (1974).

[83] M. Balkanski, K. P. Jain, R. Beserman, and M. Jouanne, Phys. Rev. B 12, 4328 (1975).

[84] M. Chandrasekhar, J. B. Renucci, and M. Cardona, Phys. Rev. B 17, 1623 (1978).

[85] K. Arya, M. A. Kanehisa, M. Jouanne, K. P. Jain, and M. Balkanski, J. Phys. C: Solid State Phys. 12, 3843 (1979).

[86] M. Chandrasekhar, H. R. Chandrasekhar, M. Grimsditch, and M. Cardona, Phys. Rev. B 22, 4825 (1980).

[87] J. Menéndez and M. Cardona, Phys. Rev. B 29, 2051 (1984).

[88] V. I. Belitsky, A. Cantarero, M. Cardona, C. Trallero-Giner, and S. T. Pavlov, J. Phys.: Condens. Matter 9, 5965 (1997).

[89] L. A. O. Nunes, L. Ioriatti, L. T. Florez, and J. P. Harbison, Phys. Rev. B 47, 13011(R) (1993).

[90] Yu. A. Pusep, M. T. O. Silva, J. C. Galzerani, S. W. da Silva, L. M. R. Scolfaro, R. Enderlein, A. A. Quivy, A. P. Lima, and J. R. Leite, Phys. Rev. B 54, 13927 (1996).

[91] K.-J. Jin, J. Zhang, Z.-H. Chen, G.-Z. Yang, Z. H. Chen, X. H. Shi, and S. C. Shen, Phys. Rev. B 64, 205203 (2001).

[92] A. E. Miroshnichenko, S. Flach, and Y. S. Kivshar, Rev. Mod. Phys. 82, 2257 (2010).

[93] Recently, there are a couple of reports regarding experimental observations of nonlinear FR in self-assembled semiconductor quantum dots and in the system of semiconductor-metal nanoparticle molecules, where strongly asymmetric spectral shapes are discerned at high cw-light power: M. Kroner, A. O. Govorov, S. Remi, B Biedermann, S. Seidl, A. Badolato, P. M. Petroff, W. Zhang, R. Barbour, B. D. Gerardot, R. J. Warburton, and K. Karrai, Nature (London) 451, 311 (2008); W. Zhang, A. O. Govorov, and G. W. Bryant, Phys. Rev. Lett. 97, 146804 (2006).

[94] T. Meier, A. Schulze, P. Thomas, H. Vaupel, and K. Maschke, Phys. Rev. B 51, 13977 (1995).

[95] U. Siegner, M.-A. Mycek, S. Glutsch, and D. S. Chemla, Phys. Rev. Lett. 74, 470 (1995).

[96] U. Siegner, M.-A. Mycek, S. Glutsch, and D. S. Chemla, Phys. Rev. B 51, 4953 (1995).

[97] K.-i. Hino, K. Goto, and N. Toshima, Phys. Rev. B 69, 035322 (2004).

[98] H. Haug and S. W. Koch, Quantum Theory of the Optical and Electronic Properties of Semiconductors, 5th ed. (World Scientific, Singapore, 2009), Chaps. 1 and 12.

[99] P. Meystre and M. Sargent III, Elements of Quantum Optics, 3rd ed. (Springer, Berlin, 1999), Chaps. 3 and 15.

[100] P. M. Morse and H. Feshbach, Methods of Theoretical Physics (McGraw-Hill, New York, 1953), Chap. 7. 
[101] N. Moiseyev, Non-Hermitian Quantum Mechanics, (Cambridge University Press, New York, 2011), Chaps. 7-9.

[102] See Supplemental Material at http://link.aps.org/supplemental/ 10.1103/PhysRevB.95.014301 for Appendix A: the solutions of eigenvalue equations (22) and (23); for Appendix B: the criterion of validity of the relation of $\left\langle\left[B_{q \alpha}, B_{q^{\prime} \alpha^{\prime}}^{\dagger}\right]\right\rangle=\delta_{q q^{\prime}} \delta_{\alpha \alpha^{\prime}}$; for Appendix $C$ : the phase-transformation invariance of the present theory; and for Appendix D: the parameters of materials and lasers employed in this study.

[103] According to Eqs. (20) and (24), a creation operator of quasiboson $B_{q \alpha}^{\dagger}(t)$ is introduced so as to ensure the commutation relation that $\left[\hat{\mathcal{H}}_{e}^{\text {(eff) }}(t), B_{q \alpha}^{\dagger}(t)\right]=B_{q \alpha}^{\dagger}(t) \mathcal{E}_{q \alpha}(t)$, where $\hat{\mathcal{H}}_{e}^{\text {(eff) }}(t)$ represents an effective electronic Hamiltonian with a correction due to the rotational-wave approximation to $\hat{\mathcal{H}}_{e}(t)$ as seen from the right-hand side of the first equality of Eq. (20); by consulting the approximations made in Sec. II A 4, the correction to $\hat{\mathcal{H}}_{e}(t)$ is given by $-\sum_{\alpha \alpha^{\prime}} B_{q \alpha}^{\dagger} U_{q \alpha}^{L \dagger} \bar{\omega} U_{q \alpha^{\prime}}^{R} B_{q \alpha^{\prime}}$. Defining the (adiabatic) ground state of $\hat{\mathcal{H}}_{e}^{\text {(eff) }}(t)$ as $|0\rangle$, one obtains the result that $\left[\hat{\mathcal{H}}_{e}^{\text {(eff) }}(t), B_{q \alpha}^{\dagger}(t)\right]|0\rangle=\left[\hat{\mathcal{H}}_{e}^{\text {(eff) }}(t)-\mathcal{E}_{0}\right]|1 ; \boldsymbol{q} \alpha\rangle=$ $\mathcal{E}_{\boldsymbol{q} \alpha}(t)|1 ; \boldsymbol{q} \alpha\rangle$, where $|1 ; \boldsymbol{q} \alpha\rangle$ is the single-quasiboson state with mode $\boldsymbol{q} \alpha$ defined by $|1 ; \boldsymbol{q} \alpha\rangle=B_{\boldsymbol{q} \alpha}^{\dagger}(t)|0\rangle$ and $\mathcal{E}_{0}$ represents the zero-point energy; setting $\mathcal{E}_{0}$ equal to zero for the sake of simplicity, one obtains the expression that $\hat{\mathcal{H}}_{e}^{(\text {eff })}(t)|1 ; \boldsymbol{q} \alpha\rangle=$ $\mathcal{E}_{\boldsymbol{q} \alpha}(t)|1 ; \boldsymbol{q} \alpha\rangle$. The above procedure of quasibosonization is reminiscent of Dyson's method of bosonization for spinwave interactions in a ferromagnet: F. J. Dyson, Phys. Rev. 102, 1217 (1956); 102, 1230 (1956). It should be noted that this quasibosonization procedure is correct just for the single-quasiboson state, and would become more questionable with increasing the number of quasibosons. For instance, it is readily shown that $\hat{\mathcal{H}}_{e}^{(\text {eff })}(t)|2 ; \boldsymbol{q} \alpha\rangle \neq$ $2 \mathcal{E}_{\boldsymbol{q} \alpha}(t)|2 ; \boldsymbol{q} \alpha\rangle$ with $|2 ; \boldsymbol{q} \alpha\rangle$ a two-quasiboson state defined as $|2 ; \boldsymbol{q} \alpha\rangle=\sqrt{2}^{-1}\left[B_{q \alpha}^{\dagger}(t)\right]^{2}|0\rangle$.

[104] Similarly to Ref. [103], the PQ creation operator $F_{q \beta}^{\dagger}(t)$ is introduced so as to ensure the commutation relation that $\left[\hat{\mathcal{H}}^{\text {(eff) }}(t), F_{q \beta}^{\dagger}(t)\right]=F_{q \beta}^{\dagger}(t) \mathcal{E}_{q \beta}(t)$, where $\hat{\mathcal{H}}^{(\text {eff })}(t)$ represents the total effective Hamiltonian given by $\hat{\mathcal{H}}^{\text {(eff) }}(t)=\hat{\mathcal{H}}_{e}^{\text {(eff) }}(t)+$
$\hat{H}_{p}+\hat{H}_{e p}$, and the expression $h_{\boldsymbol{q}}(t)=\sum_{\beta} V_{\boldsymbol{q} \beta}^{R}(t) \mathcal{E}_{\boldsymbol{q} \beta}(t) V_{\boldsymbol{q} \beta}^{L \dagger}(t)$ is used in view of Eq. (39).

[105] W. Schäfer and M. Wegener, Semiconductor Optics and Transport Phenomena (Springer, Berlin, 2002), Chaps. 2, 10, and 11.

[106] A. L. Fetter and J. D. Walecka, Quantum Theory of ManyParticle Systems (McGraw-Hill, New York, 1971), Chaps. 3-5.

[107] R. Binder, D. Scott, A. E. Paul, M. Lindberg, K. Henneberger, and S. W. Koch, Phys. Rev. B 45, 1107 (1992).

[108] P. Y. Yu and M. Cardona, Fundamentals of Semiconductors, 4th ed. (Springer, Berlin, 2010), Chaps. 3 and 7.

[109] B. W. Shore, Rev. Mod. Phys. 39, 439 (1967).

[110] T. Inui, Y. Tanabe, and Y. Onodera, Group Theory and Its Applications in Physics, Springer Series in Solid-State Sciences, Vol. 78 (Springer, Berlin, 1990), Chaps. 11 and 12.

[111] H. W. K. Tom, T. F. Heinz, and Y. R. Shen, Phys. Rev. Lett. 51, 1983 (1983).

[112] P. Stampfli and K. H. Bennemann, Phys. Rev. B 49, 7299 (1994).

[113] The calculated results are the first success in showing in a fully quantum-mechanical manner the manifestation of the transient FR due to the CP generation in Si. Nevertheless, the close comparison with the experimental results seems limited because the excitation carrier densities for both results are markedly different each other; the maximum density in the calculation is $6.31 \times 10^{17} \mathrm{~cm}^{-3}$, as shown in Sec. III A, while the average density in the experiment is $4 \times 10^{19} \mathrm{~cm}^{-3}$. With the increase in the excitation carrier density, it is considered that the nonadiabatic interaction is more significant, leading to the alteration of spectral profiles; this interaction is neglected in the present framework, as discussed in Sec. II A 4. Actually, the sign of the asymmetry parameter for the FR spectra of Figs. 3(b) and 6(b) is positive, while that for the experimental spectra is negative. According to our preliminary evaluation incorporating the nonadiabatic interaction, it is found that the spectral profile of FR varies due to this effect, and the sign of the asymmetry likely changes for the interaction stronger, though not shown here.

[114] M. Hase, Appl. Phys. Lett. 94, 112111 (2009). 